\title{
ON THE NUMBER OF SOLUTIONS OF POLYNOMIAL CONGRUENCES AND THUE EQUATIONS
}

\author{
C. L. STEWART
}

\section{INTRODUCTION}

Let $F(x, y)=a_{r} x^{r}+a_{r-1} x^{r-1} y+\cdots+a_{0} y^{r}$ be a binary form with rational integer coefficients and with $r \geq 3$. Let $h$ be a nonzero integer. In 1909 Thue proved that if $F$ is irreducible then the equation

$$
F(x, y)=h
$$

has only finitely many solutions in integers $x$ and $y$. In the first part of this paper we shall establish upper bounds for the number of solutions of (1) in coprime integers $x$ and $y$ under the assumption that the discriminant $D(F)$ of $F$ is nonzero. For most integers $h$ these bounds improve upon those obtained by Bombieri and Schmidt in [5]. In the course of proving these bounds we shall establish a result on polynomial congruences that extends earlier work of Nagell [30], Ore [32], Sándor [33], and Huxley [19]. In fact we shall establish an upper bound for the number of solutions of a polynomial congruence that is, in general, best possible.

In the second part we shall address the problem of finding forms $F$ for which (1) has many solutions for arbitrarily large integers $h$. Finally we shall obtain upper bounds for the number of solutions of certain Thue-Mahler and Ramanujan-Nagell equations by appealing to estimates of Evertse, Györy, Stewart, and Tijdeman [17] for the number of solutions of $S$-unit equations.

\section{The Thue And Thue-Mahler equations}

For any nonzero integer $h$ let $\omega(h)$ denote the number of distinct prime factors of $h$. In 1933 Mahler [23] proved that if $F$ is irreducible then (1) has at most $C_{1}^{1+\omega(h)}$ solutions in coprime integers $x$ and $y$, where $C_{1}$ is a positive number that depends on $F$ only. Let $p_{1}, \ldots, p_{t}$ be distinct prime numbers. The equation

$$
F(x, y)=p_{1}^{k_{1}} \cdots p_{t}^{k_{t}}
$$

Received by the editors March 21, 1991.

1991 Mathematics Subject Classification. Primary 11D41, 11C08, 11J25.

$K e y$ words and phrases. Thue equations, polynomial congruences, $S$-unit equations.

Research supported in part by a Killam Research Fellowship and by Grant A3528 from the Natural Sciences and Engineering Research Council of Canada. 
in coprime integers $x$ and $y$ and integers $k_{1}, \ldots, k_{t}$ is known as a ThueMahler equation. In fact Mahler proved the stronger result that (2) has at most $C_{1}^{1+t}$ such solutions. In 1938 Erdös and Mahler [9] proved that if $F$ has nonzero discriminant, $h>C_{2}$ and $g$ is a divisor of $h$ with $g>h^{6 / 7}$ then the number of solutions of (1) in coprime integers $x$ and $y$ is at most $C_{3}^{1+\omega(g)}$, where $C_{2}$ and $C_{3}$ are positive numbers that depend on $F$ only. In 1961 Lewis and Mahler [21] showed that the number of primitive solutions of (2), that is, solutions with $x$ and $y$ coprime, is at most

$$
c_{1}(a r)^{c_{2} \sqrt{r}}+\left(c_{3} r\right)^{1+t}
$$

where $c_{1}, c_{2}$, and $c_{3}$ are absolute constants, provided $F$ has nonzero discriminant, $a_{r} a_{0} \neq 0$, and the coefficients of $F$ have absolute values not exceeding $a$. In 1984 Evertse [13] gave

$$
2 \cdot 7^{r^{3}(2 t+3)}
$$

as an upper bound for the number of primitive solutions of (2) under the assumption that $F$ is divisible by at least three pairwise linearly independent linear forms in some algebraic number field. Evertse's result resolved a conjecture of Siegel since his upper bound for the number of primitive solutions of (2) depends only on $r$ and $t$, and so for (1) depends only on $r$ and $\omega(h)$, and does not depend on the coefficients of $F$. In 1987 Bombieri and Schmidt [5] refined the result of Evertse for the Thue equation. They proved that if $F$ is irreducible then the number of solutions of (1) in coprime integers $x$ and $y$ is at most

$$
c_{4} r^{1+\omega(h)},
$$

where $c_{4}$ is an absolute constant. Further they showed that one may take $c_{4}$ to be 430 if $r$ is sufficiently large.

Let

$$
A=\left(\begin{array}{ll}
a & b \\
c & d
\end{array}\right)
$$

and define the binary form $F_{A}$ by

$$
F_{A}(x, y)=F(a x+b y, c x+d y) \text {. }
$$

Observe that if $A$ is in $\operatorname{GL}(2, \mathbb{Z})$, in other words, $A$ has integer entries and determinant \pm 1 , and $(x, y)$ is a solution of (1) in coprime integers $x$ and $y$ then $A(x, y)=(a x+b y, c x+d y)$ is a solution of $F_{A^{-1}}(X, Y)=h$ in coprime integers. For any $A \in \mathrm{GL}(2, \mathbb{Z})$ we say that $F_{A}$ and $-F_{A}$ are equivalent to $F$. We remark that the number of solutions of $(1)$ in coprime integers is the same for equivalent forms. For any polynomial $G$ in $\mathbb{C}\left[z_{1}, \ldots, z_{n}\right]$ that is not identically zero the Mahler measure $M(G)$ is defined by

$$
M(G)=\exp \int_{0}^{1} d t_{1} \ldots \int_{0}^{1} d t_{n} \log \left|G\left(e^{2 \pi i t_{1}}, \ldots, e^{2 \pi i t_{n}}\right)\right|
$$


Thus if $n=1$ and $G(z)=a_{r}\left(z-\alpha_{1}\right) \cdots\left(z-\alpha_{r}\right)$ with $a_{r} \neq 0$, then, by Jensen's theorem,

$$
M(G)=\left|a_{r}\right| \prod_{i=1}^{r} \max \left(1,\left|\alpha_{i}\right|\right)
$$

Suppose that $F$ is a binary form that factors as $\prod_{j=1}^{r}\left(\alpha_{j} x-\beta_{j} y\right)$. The discriminant $D=D(F)$ of $F$ is given by

$$
D(F)=\prod_{i<j}\left(\alpha_{i} \beta_{j}-\alpha_{j} \beta_{j}\right)^{2} .
$$

For any nonzero integer $t$ we have

$$
D(t F)=t^{2(r-1)} D(F),
$$

and for any matrix $A$ with integer entries

$$
D\left(F_{A}\right)=(\operatorname{det} A)^{r(r-1)} D(F) .
$$

Thus for any $A \in \mathrm{GL}(2, \mathbb{Z})$ we have $D=D(F)=D\left(F_{A}\right)$. For any nonzero integer $n$ and prime number $p$ let $\operatorname{ord}_{p} n$ denote the exact power of $p$ that divides $n$. For any real number $x$ let $[x]$ denote the greatest integer less than or equal to $x$. Let $p$ be a prime number, and let $r, k$, and $D$ be integers with $r \geq 2$ and $D \neq 0$. We define $T=T(r, k, p, D)$ by

$$
T=\min \left(\left[\left(\frac{r-1}{r}\right) k\right], \min _{j=0, \ldots, r-2}\left(\left[\frac{\operatorname{ord}_{p} D}{(j+1)(j+2)}+\left(\frac{j}{j+2}\right) k\right]\right)\right)
$$

and for any nonzero integer $g$ we define $G(g, r, D)$ by

$$
G(g, r, D)=\prod_{p \mid g} p^{T\left(r, \operatorname{ord}_{p} g, p, D\right)} .
$$

Recall that the content of $F$ is the greatest common divisor of the coefficients of $F$. We shall prove the following result.

Theorem 1. Let $F$ be a binary form with integer coefficients of degree $r(\geq 3)$, content 1 , and nonzero discriminant $D$. Let $h$ be a nonzero integer, and let $\varepsilon$ be a positive real number. Let $g$ be any divisor of $h$ with

$$
\frac{g^{1+\varepsilon}|D|^{1 / r(r-1)}}{G(g, r, D)} \geq|h|^{2 / r+\varepsilon} \text {. }
$$

The number of pairs of coprime integers $(x, y)$ for which $F(x, y)=h$ is at most

$$
2800\left(1+\frac{1}{8 \varepsilon r}\right) r^{1+\omega(g)}
$$

Notice that if $g$ or $D$ is squarefree, or if $g$ and $D$ are coprime, then $G(g, r, D)$ is 1 . Further $G(g, r, D)$ is always bounded from above by $\left(D, g^{2}\right)^{1 / 2}$; here $\left(D, g^{2}\right)$ denotes the greatest common divisor of $D$ and $g^{2}$. Thus Theorem 1 sharpens the result of Erdös and Mahler [9]. Furthermore if 
we take $g=|h|$ and $\varepsilon$ any positive real number then condition (8) holds since on taking $j=r-2$ in (7) we see that

$$
T(r, k, p, D) \leq\left[\frac{\operatorname{ord}_{p} D}{(r-1) r}+\left(\frac{r-2}{r}\right) k\right],
$$

hence

$$
G(|h|, r, D) \leq|h|^{(r-2) / r}|D|^{1 / r(r-1)} .
$$

Thus if $D(F) \neq 0$ then the number of primitive solutions of (1) is at most $2800 r^{1+\omega(h)}$; in particular, we recover estimate (4) of Bombieri and Schmidt. In general this choice for $g$ and $\varepsilon$ is not optimal. Indeed the significant feature of estimate (9) is that the term $\omega(h)$ in estimate (4) has been replaced by the quantity $\omega(g)$. For almost all integers $h$ in the sense of natural asymptotic density, and any $\delta>0, \omega(h)=\log \log h+O\left(\log \log h^{1 / 2+\delta}\right)$ (see [18]). On the other hand (see [6]), if $\varepsilon<(r-2) / r$ then for a positive proportion of integers $h$ we may take $g$ to be a prime, hence $\omega(g)=1$, and estimate (9) becomes $C(\varepsilon) r^{2}$. In fact $\omega(h)$ may be as large as $\log h /(4 \log \log h)$ while $\omega(g)=1$. No particular significance attaches to the constant 2800 in (9). It can certainly be improved. In particular, if either $h$ or $r$ is large, then (9) holds with a much smaller constant.

Our proof depends upon the Thue-Siegel principle as enunciated in Bombieri and Schmidt [5] and follows quite closely the proof given in [5]. (The author would like to thank Professor Evertse for his suggestion, in connection with an earlier version of this result, that he follow the approach of Bombieri and Schmidt [5] for dealing with the small solutions of (1). This allowed him to remove a factor involving $M(F)$ from his original estimates.)

Our argument differs from that of Bombieri and Schmidt in that they reduce the study of (1) to the case when $h=1$ by splitting solutions according to congruence classes modulo $h$. On the other hand, we reduce $h$ to $h / g$ by splitting the solutions into congruence classes modulo $g$. Further we appeal to Theorem 2 to spread apart solutions in the same congruence class. Both arguments owe much to the work of Mahler [26].

Observe that if $|D|^{1 / r(r-1)} \geq|h|^{2 / r+\varepsilon}$, then we may apply Theorem 1 with $g=1$ to deduce that the number of pairs of coprime integers $(x, y)$ for which (1) holds is at most

$$
2800\left(1+\frac{1}{8 \varepsilon r}\right) r .
$$

Evertse and Györy $[12,16]$ have obtained a related result for the Thue inequality

$$
0<|F(x, y)| \leq h .
$$

Define $(N(r), \delta(r))$ by $(N(r), \delta(r))=\left(6 r 7^{2\left(\begin{array}{c}r \\ 3\end{array}\right)}, \frac{5}{6} r(r-1)\right)$ for $3 \leq r<400$ and $(N(r), \delta(r))=(6 r, 120(r-1))$ for $r>400$. They prove that if

$$
|D| \geq h^{\delta(r)} \exp (80 r(r-1)) \text {, }
$$


then the number of solutions of (10) in coprime integers $x$ and $y$ with $y$ positive is at most $N(r)$.

Recall that the term in the denominator on the left-hand side of inequality (8) is at most $\left(D, g^{2}\right)^{1 / 2}$. If $g \geq|h|^{2 / r+\varepsilon}$ then, since $|D|$ is at least 1 , whenever $\left(D, g^{2}\right)^{1 / 2} \leq|h|^{\varepsilon / 2}$ inequality (8) holds with $\varepsilon$ replaced by $\varepsilon / 2$. This gives immediately the following consequence of Theorem 1 .

Corollary 1. Let $F$ be a binary form with integer coefficients of degree $r(\geq 3)$, content 1 , and nonzero discriminant $D$. Let $h$ be a nonzero integer and let $\varepsilon$ be a positive real number. Let $g$ be any divisor of $h$ with $g \geq|h|^{2 / r+\varepsilon}$. If $|h| \geq\left(D, g^{2}\right)^{1 / \varepsilon}$ then the number of pairs of coprime integers $(x, y)$ for which $F(x, y)=h$ is at most

$$
2800\left(1+\frac{1}{4 \varepsilon r}\right) r^{1+\omega(g)} .
$$

If $F$ has few nonzero coefficients, say $s$, then upper bounds for the number of primitive solutions of (1) have been given by Mueller and Schmidt [29] and Schmidt [34] that depend on $s$ and $h$ only. Further, the special case of binomial forms $F(x, y)=a_{r} x^{r}+a_{0} y^{r}$ has been much studied by the hypergeometric method. This study was initiated by Siegel [35] in 1937 and refined by several authors, most recently Evertse [11] in 1982; see, in particular, Theorem 2 of [11], which is of a similar character to Corollary 1. Finally we mention that Silverman [36] in 1983 proved that if $D(F) \neq 0$ and $h$ is $r$-powerfree and sufficiently large relative to $F$ then the number of primitive solutions of (1) is at most

$$
r^{2 r^{2}}\left(8 r^{3}\right)^{R_{F}(h)}
$$

where $R_{F}(h)$ is the rank of the Mordell-Weil group of the Jacobian of the curve (1) over $\mathbb{Q}$.

\section{ON POLYNOMIAL CONGRUENCES}

The results in this section were motivated by the reduction theory of $\S \mathrm{VI}$ of Bombieri and Schmidt [5]. The author is grateful to Professor Bombieri for correspondence that clarified for him some aspects of their argument.

Let $\Omega_{p}$ be a completion of an algebraic closure of $\mathbb{Q}_{p}$, the field of $p$-adic numbers. Let ||$_{p}$ denote the usual $p$-adic value in $\mathbb{Q}_{p}$, so $|p|_{p}=p^{-1}$, as well as an extension of it to $\Omega_{p}$. Let $\mathbb{Z}_{p}$ denote the ring of integers in $\mathbb{Q}_{p}$, and let $R_{p}$ denote the ring of elements $\alpha$ in $\Omega_{p}$ with $|\alpha|_{p} \leq 1$. We define ord $\gamma$ for $\gamma \in \Omega_{p}$ by $\operatorname{ord}_{p} \gamma=-\left(\log |\gamma|_{p}\right) / \log p$. Recall that the content of a polynomial $f$ with integer coefficients is the greatest common divisor of its coefficients and the discriminant $D(f)$ of $f$ is given by $D(F)$, where $F$ is the binary form $F(x, y)=y^{r} f(x / y)$ and $r$ is the degree of $f$.

For any prime $p$ and nonzero integer $D$ we define $l=l(p, D)$ by

$$
l=\operatorname{ord}_{p} D .
$$


Further for primes $p$ and nonzero integers $r, k$, and $D$ with $r \geq 2$, we have that $T=T(r, k, p, D)$, as defined in (7), satisfies

$$
T= \begin{cases}{\left[\frac{l}{2}\right]} & \text { if } k \geq l, \\ {\left[\frac{l}{(j+1)(j+2)}+\left(\frac{j}{j+2}\right) k\right]} & \text { if } \frac{l}{j} \geq k \geq \frac{l}{j+1} \text { for } j=1, \ldots, r-2, \\ {\left[\left(\frac{r-1}{r}\right) k\right]} & \text { if } \frac{l}{r-1} \geq k \geq 1\end{cases}
$$

Theorem 2. Let $p$ be a prime number, and let $f$ be a polynomial with integer coefficients, content coprime with $p$, degree $r(\geq 2)$, and nonzero discriminant $D$. Put $\operatorname{ord}_{p} D=l$ and let $s$ denote the number of zeros of $f$ in $R_{p}$. For each positive integer $k$ there is an integer $t \quad(=t(k))$ with $0 \leq t \leq s \quad(\leq r)$ and there are nonnegative integers $b_{1}\left(=b_{1}(k)\right), \ldots, b_{t}\left(=b_{t}(k)\right)$ and $u_{1}$ $\left(=u_{1}(k)\right), \ldots, u_{t}\left(=u_{t}(k)\right)$ such that the complete solution of the congruence

$$
f(x) \equiv 0 \quad\left(\bmod p^{k}\right),
$$

is given by the $t$ congruences

$$
x \equiv b_{i} \quad\left(\bmod p^{k-u_{i}}\right)
$$

for $i=1, \ldots, t$ and such that if $k>l$ then $t(k)=t(l+1)$ and $u_{i}(k)=u_{i}(l+1)$ for $i=1, \ldots, t$, while if $j \geq k>l$ then

$$
b_{i}(j) \equiv b_{i}(k) \quad\left(\bmod p^{k-u_{i}(k)}\right)
$$

for $i=1, \ldots, t$. Further, for each positive integer $k, p^{k}$ divides the content of $f\left(p^{k-u_{i}} x+b_{i}\right)$ for $i=1, \ldots, t$,

$$
0 \leq u_{i}(k) \leq T
$$

for $i=1, \ldots, t$, and

$$
u_{1}+\cdots+u_{t} \leq \min \left(2\left[\frac{l}{2}\right], r\left[\left(\frac{r-1}{r}\right) k\right]\right) .
$$

Furthermore, for each positive integer $k$, at most $s_{1}$ of the integers $b_{1}, \ldots, b_{t}$ are divisible by $p$, where $s_{1}$ is the number of roots $\alpha$ of $f$ with $|\alpha|_{p}<1$.

Proof of Theorem 2. We first prove that the solutions of (11) are given by $t$ such congruences and to this end our argument will follow initially that of Lemma 7 of [5] or Proposition 4.8.1 of [1]. Let $U=\left\{\left.\sigma \in R_{p}|| f(\sigma)\right|_{p} \leq p^{-k}\right\}$. Then $U$ can be written as the disjoint union of maximal discs in $R_{p}$. Let $a R_{p}+b$ be such a disc with $a, b \in R_{p}, a \neq 0$, and $|a|_{p} \leq|b|_{p}$. Since $f$ has content coprime with $p$ there exists a $\gamma$ in $R_{p}$ with $|f(\gamma)|_{p}=1$. Thus $a R_{p}+b$ is properly contained in $R_{p}$ hence $|a|_{p}<1$. Now let $f(x)=a_{r}\left(x-\alpha_{1}\right) \cdots\left(x-\alpha_{r}\right)$ in $\Omega_{p}$. Observe now that since $a R_{p}+b$ is maximal, it contains a root $\alpha_{i}$ of 
$f$. For otherwise there exists an $a^{\prime} \in R_{p}$ with $|a|_{p}<\left|a^{\prime}\right|_{p}<\left|\alpha_{i}-b\right|_{p}$ for $i=1, \ldots, r$ and so $a^{\prime} R_{p}+b$ is a disc in $U$ that properly contains $a R_{p}+b$, contradicting the assumption that $a R_{p}+b$ is maximal. Thus each maximal disc contains a root of $f$ in $R_{p}$ and so $U$ is the disjoint union of at most $s$ such discs. For each disc $a R_{p}+b$ we consider the disc $a R_{p}+b \cap \mathbb{Z}_{p}$. This disc is either empty or of the form $A \mathbb{Z}_{p}+B$ with $A, B \in \mathbb{Z}_{p},|A|_{p} \leq|B|_{p}$, and $|A|_{p}<1$ since $|a|_{p}<1$. If $x$ in $\mathbb{Z}$ satisfies $f(x) \equiv 0\left(\bmod p^{k}\right)$ then $|f(x)|_{p} \leq p^{-k}$ and so $x$ lies in one of the discs $A \mathbb{Z}_{p}+B$. Thus there exists an integer $t$ with $0 \leq t \leq s$ and integers $b_{1}, \ldots, b_{t}$ and $u_{1}, \ldots, u_{t}$ with $0 \leq u_{i} \leq k$ for $i=1, \ldots, t$ such that $x$ satisfies one of the congruences $x \equiv b_{i}\left(\bmod p^{k-u_{i}}\right)$ with $1 \leq i \leq t$.

For each integer $i$ for which $k-u_{i}$ is less then $k$ we consider the integers $e_{i, j}=b_{i}+j p^{k-u_{i}}$ for $j=1, \ldots, p$. If for some integer $i$ and for each integer $j$ from 1 to $p$ there is a root of $f$, say $\alpha$, for which $\left|\alpha-e_{i, j}\right|_{p}<\left|\alpha-e_{i, m}\right|_{p}$ for $1 \leq m \leq p$ with $m \neq j$ then we replace the single congruence $x \equiv b_{i}$ $\left(\bmod p^{k-u_{i}}\right)$ by the $p$ congruences $x \equiv e_{i, j}\left(\bmod p^{k-u_{i}+1}\right)$ for $j=1, \ldots, p$. We now relabel the $b_{i}$ 's and $u_{i}$ 's to take into account the fact that we have $p$ new congruences in place of the single congruence $x \equiv b_{i}\left(\bmod p^{k-u_{i}}\right)$. Since each maximal disc contains a root of $f$ we see that to each of the original $b_{i}$ 's we may associate a root of $f$ that is $p$-adically closer to it than to any of the other $b_{i}$ 's. This situation still applies after the above substitution. Each such root is one of the $s$ roots of $f$ from $R_{p}$ and hence $0 \leq t \leq s$. Further, there can be only finitely many applications of the above procedure. Thus, we may assume that for each integer $i$ for which $k-u_{i}$ is less than $k$ there is an integer $j=j(i)$ with $1 \leq j \leq p$ such that for each root $\alpha$ of $f$ there is an integer $m=m(\alpha, j)$, different from $j$, with $1 \leq m \leq p$ such that $\left|\alpha-e_{i, m}\right|_{p} \leq\left|\alpha-e_{i, j}\right|_{p}$. But $\left|e_{i, j}-e_{i, m}\right|_{p}=p^{-k+u_{i}}$ and so by the triangle inequality $\left|\alpha-e_{i, j}\right|_{p} \geq p^{-k+u_{i}}$. Note that we may replace $b_{i}$ by $e_{i, j}$ and so may suppose that $\left|\alpha-b_{i}\right|_{p} \geq p^{-k+u_{i}}$ for all roots $\alpha$ of $f$ whenever $k-u_{i}<k$. Then we may suppose, without loss of generality, that $u_{i}>0$ for $i=1, \ldots, t_{1}$, and that $u_{i}=0$ for $i=t_{1}+1, \ldots, t$, where $t_{1}$ is an integer with $0 \leq t_{1} \leq t$. Further, again without loss of generality, we may suppose that the roots of $f$ are ordered so that

$$
\left|\alpha_{i}-b_{i}\right|_{p} \leq\left|\alpha_{j}-b_{i}\right|_{p}
$$

for $i=1, \ldots, t$ and $j=1, \ldots, r$. Put

$$
\delta_{i, j}=\operatorname{ord}_{p}\left(b_{i}-\alpha_{j}\right)
$$

for $i=1, \ldots, t$ and $j=1, \ldots, r$ and note that, by (16),

$$
\delta_{i, i} \geq \delta_{i, j}
$$


for $i=1, \ldots, t$ and $j=1, \ldots, r$. Since $\left|f\left(b_{i}\right)\right|_{p} \leq p^{-k}$ we have

$$
\operatorname{ord}_{p} a_{r}+\sum_{j=1}^{r} \delta_{i, j} \geq k
$$

for $i=1, \ldots, t$. Also, since $\left|\alpha_{j}-b_{i}\right|_{p} \geq p^{-k+u_{i}}$ we have

$$
\delta_{i, j} \leq k-u_{i}
$$

for $i=1, \ldots, t_{1}$ and $j=1, \ldots, r$. Since $f$ has content coprime with $p$,

$$
\operatorname{ord}_{p} a_{r}+\sum_{\operatorname{ord}_{p} \alpha_{j}<0} \operatorname{ord}_{p} \alpha_{j}=0
$$

Thus, for all integers $b$,

$$
\operatorname{ord}_{p} a_{r}+\sum_{\operatorname{ord}_{p} \alpha_{j}<0} \operatorname{ord}_{p}\left(b-\alpha_{j}\right)=0 .
$$

Accordingly, by (18) and (21) with $b$ replaced by $b_{i}$,

$$
\sum_{1 \leq j \leq r, \delta_{i, j} \geq 0} \delta_{i, j} \geq k
$$

for $i=1, \ldots, t$. Therefore, by (19) and (22),

$$
\sum_{1 \leq j \leq r, j \neq i}^{*} \delta_{i, j} \geq u_{i}
$$

for $i=1, \ldots, t$. Here $\sum^{*}$ indicates that the sum is taken over those terms $\delta_{i, j}$ that are nonnegative. Further, for integers $i$ and $j$ with $1 \leq i<j \leq r$, $\alpha_{i}-\alpha_{j}=\left(b_{h}-\alpha_{j}\right)-\left(b_{h}-\alpha_{i}\right)$ for $h=1, \ldots, t$, hence

$$
\operatorname{ord}_{p}\left(\alpha_{i}-\alpha_{j}\right) \geq \max _{1 \leq h \leq t}\left\{\min \left(\delta_{h, i}, \delta_{h, j}\right)\right\}
$$

Recall that

$$
\frac{l}{2}=\frac{1}{2} \operatorname{ord}_{p} D=(r-1) \operatorname{ord}_{p} a_{r}+\sum_{i<j} \operatorname{ord}_{p}\left(\alpha_{i}-\alpha_{j}\right) .
$$

By (20),

$$
(r-1) \operatorname{ord}_{p} a_{r}+\sum_{i<j, \min \left(\operatorname{ord}_{p} \alpha_{i}, \operatorname{ord}_{p} \alpha_{j}\right)<0} \operatorname{ord}_{p}\left(\alpha_{i}-\alpha_{j}\right) \geq 0
$$

and so, by (25),

$$
\frac{l}{2} \geq \sum_{i<j, \min \left(\operatorname{ord}_{p} \alpha_{i}, \operatorname{ord}_{p} \alpha_{j}\right) \geq 0} \operatorname{ord}_{p}\left(\alpha_{i}-\alpha_{j}\right) .
$$

Thus it follows from (24) that

$$
\frac{l}{2} \geq \sum_{i<j}^{*} \max _{1 \leq h \leq t}\left\{\min \left(\delta_{h, i}, \delta_{h, j}\right)\right\} .
$$


By $(17), \min \left(\delta_{i, i}, \delta_{i, j}\right)=\delta_{i, j}$ for $i \neq j$ and so

$$
\frac{l}{2} \geq \sum_{1 \leq j \leq r, j \neq i}^{*} \delta_{i, j}+\sum_{j<m, j \neq i, m \neq i}^{*} \min \left(\delta_{i, j}, \delta_{i, m}\right)
$$

for $i=1, \ldots, t$.

Let us for the moment fix $i$ with $1 \leq i \leq t$, and put $u_{i}=u$. Let $n$ denote the number of terms $\delta_{i, j}$ with $i \neq j$ and $\delta_{i, j} \geq 0$; note that $0 \leq n \leq r-1$. Relabel these terms as $x_{1}, \ldots, x_{n}$ in such a way that $x_{1} \geq x_{2} \geq \cdots \geq x_{n} \geq 0$. Then, from (28),

$$
\frac{l}{2} \geq x_{1}+\cdots+x_{n}+\sum_{1 \leq j<m \leq n} \min \left(x_{j}, x_{m}\right),
$$

hence

$$
\frac{l}{2} \geq x_{1}+2 x_{2}+\cdots+n x_{n}
$$

Further, by (23),

$$
x_{1}+\cdots+x_{n} \geq u
$$

and, by (19),

$$
-x_{m} \geq u-k
$$

for $m=1, \ldots, n$. Since $x_{m} \geq 0$ for $m=1, \ldots, n$ we deduce from (29) that

$$
\frac{l}{2} \geq j\left(x_{1}+\cdots+x_{n}\right)-(j-1) x_{1}-(j-2) x_{2}-\cdots-x_{j-1}
$$

for $j=1, \ldots, n$. By (30) and (31),

$$
\frac{l}{2} \geq j u+\frac{j(j-1)}{2}(u-k)
$$

hence

$$
u \leq \frac{l}{j(j+1)}+\left(\frac{j-1}{j+1}\right) k
$$

for $j=1, \ldots, n$. It also follows from (30) and (31) that $n(k-u) \geq u$, whence

$$
u \leq\left(\frac{n}{n+1}\right) k \text {. }
$$

Therefore

$$
u \leq \min \left(\left(\frac{n}{n+1}\right) k, \min _{j=0, \ldots, n-1}\left(\frac{l}{(j+1)(j+2)}+\left(\frac{j}{j+2}\right) k\right)\right) .
$$

Since $n \leq r-1$, we certainly have

$$
u \leq \min \left(\left(\frac{r-1}{r}\right) k, \min _{j=0, \ldots, r-2}\left(\frac{l}{(j+1)(j+2)}+\left(\frac{j}{j+2}\right) k\right)\right),
$$

which establishes (14). 
Note that for any pair of integers $(i, j)$ with $1 \leq i<j \leq t$,

(32) $\max _{1 \leq h \leq t}\left(\min \left(\delta_{h, i}, \delta_{h, j}\right)\right) \geq \frac{\min \left(\delta_{i, i}, \delta_{i, j}\right)+\min \left(\delta_{j, i}, \delta_{j, j}\right)}{2} \geq \frac{\delta_{i, j}+\delta_{j, i}}{2}$.

Further, for any pair of integers $(i, j)$ with $1 \leq i \leq t<j \leq r$,

$$
\max _{1 \leq h \leq t}\left(\min \left(\delta_{h, i}, \delta_{h, j}\right)\right) \geq \delta_{i, j} \text {. }
$$

Thus, by (27),

$$
l \geq \sum_{i=1}^{t}\left(\sum_{1 \leq j \leq r, j \neq i}^{*} \delta_{i, j}+\sum_{t<j \leq r}^{*} \delta_{i, j}\right)
$$

and, by (23),

$$
u_{1}+\cdots+u_{t} \leq l
$$

Observe that if (34) holds with equality then (23), (27), and (33) must also hold with equality. By (23), $\delta_{i, j} \leq 0$ whenever $i>t_{1}$. By (33), if $1 \leq i \leq t_{1}$ and $t<j \leq r$ then $\delta_{i, j} \leq 0$. By (32), if $1 \leq i \leq t, i<j \leq t$, and $\delta_{i, j} \geq 0$ then $\delta_{i, j}=\delta_{j, i}$. Therefore if $u_{1}+\cdots+u_{t}=l$ then, by (33),

$$
l=\sum_{i=1}^{t_{1}}\left(\sum_{1 \leq j \leq t, j \neq i}^{*} \delta_{i, j}\right)=\sum_{i=1}^{t_{1}} 2\left(\sum_{i<j \leq t_{1}}^{*} \delta_{i, j}\right) .
$$

Further if (34) holds with equality then by (19) and (23) we see that $\delta_{i, i}=$ $k-u_{i}$ for $i=1, \ldots, t_{1}$. Since

$$
\left(\alpha_{i}-b_{i}\right)-\left(\alpha_{i}-b_{j}\right)=b_{j}-b_{i}
$$

and

$$
\left|\alpha_{i}-b_{i}\right|_{p} \leq\left|\alpha_{i}-b_{j}\right|_{p}
$$

for $1 \leq i \leq t_{1}$ and $1 \leq j \leq r$ we deduce that

$$
\left|\alpha_{i}-b_{j}\right|_{p}=\max \left(\left|\alpha_{i}-b_{i}\right|_{p},\left|b_{j}-b_{i}\right|_{p}\right) \text {. }
$$

Since $\delta_{i, i}$ is an integer and $\operatorname{ord}_{p}\left(b_{i}-b_{j}\right)$ is also an integer, we conclude that $\delta_{i, j}$ is an integer for all pairs $(i, j)$ with $1 \leq i \leq t_{1}$ and $1 \leq j \leq r$. Therefore, by $(35) ; l$ is an even integer. Accordingly, when $l$ is odd inequality (34) is not sharp and so we may replace $l$ in (34) by $l-1$. Therefore we have

$$
u_{1}+\cdots+u_{t} \leq 2\left[\frac{l}{2}\right] \text {. }
$$

Further since $t \leq r$ and $T \leq[((r-1) / r) k]$ we also have

$$
u_{1}+\cdots+u_{t} \leq r\left[\left(\frac{r-1}{r}\right) k\right] \text {. }
$$

Next consider $f\left(p^{k-u_{i}} x+b_{i}\right)$ for $i=1, \ldots, t$. If $t_{1}+1 \leq i \leq t$ then $u_{i}=0$ and since $f\left(b_{i}\right) \equiv 0\left(\bmod p^{k}\right)$ it is immediate that $p^{k}$ divides the 
content of $f$. Suppose therefore that $1 \leq i \leq t_{1}$, hence that $u_{i}>0$.We have $\left|p^{-k} f\left(p^{k-u_{i}} x+b_{i}\right)\right|_{p} \leq 1$ for all $x$ in $\mathbb{Z}_{p}$ and it suffices to prove that $\left|p^{-k} f\left(p^{k-u_{i}} x+b_{i}\right)\right|_{p} \leq 1$ for all $x$ in $R_{p}$. We have

$$
\left|f\left(p^{k-u_{i}} x+b_{i}\right)\right|_{p}=\left|a_{r}\right|_{p} \prod_{j=1}^{r}\left|p^{k-u_{i}} x+b_{i}-\alpha_{j}\right|_{p} .
$$

Recall that $\left|b_{i}-\alpha_{j}\right|_{p} \geq p^{-\left(k-u_{i}\right)}$ for $j=1, \ldots, r$. Thus for $x$ in $R_{p}$, $\left|p^{k-u_{i}} x+b_{i}-\alpha_{j}\right|_{p} \leq\left|b_{i}-\alpha_{j}\right|_{p}$ for $j=1, \ldots, r$ and so for all $x$ in $R_{p}$, $\left|p^{-k} f\left(p^{k-u_{i}} x+b_{i}\right)\right|_{p} \leq\left|p^{-k} f\left(b_{i}\right)\right|_{p} \leq 1$, as required.

Next we take $k=l+1$ in (11) and apply the argument of Sándor [33] to lift the $t=t(l+1)$ congruences (12). This then gives that for $k>l, t(k)=t(l+1)$ and $u_{i}(k)=u_{i}(l+1)$ for $i=1, \ldots, t$ and also yields (13).

Finally, observe that if $p \mid b_{i}$ for some $i$ with $1 \leq i \leq t$ then $b_{i} \in A \mathbb{Z}_{p}+B$ for one of the discs with $|B|_{p} \leq p^{-1}$. Therefore the maximal disc $a R_{p}+b$ for which $a R_{p}+b \cap \mathbb{Z}_{p}=A \mathbb{Z}_{p}+B$ satisfies $|b|_{p}<1$. Since $a R_{p}+b$ is maximal it contains a root $\alpha$ of $f$ and since $|a|_{p} \leq|b|_{p},|\alpha|_{p}<1$. Therefore at most $s_{1}$ of the integers $b_{1}, \ldots, b_{t}$ are divisible by $p$. This complete the proof.

That we may take $t(k)$ and $u_{i}(k)$ for $i=1, \ldots, t$ to be constant for $k>l$ follows from an argument of Sándor [33] as does the fact that the condition $k>l$ cannot be weakened. It follows from Lemma 7 of Bombieri and Schmidt [5] that $p^{k}$ divides the content of $f\left(p^{k-u_{i}}+b_{i}\right)$ for $i=1, \ldots, t$. However, they do not give an estimate for $u_{i}$. Indeed, the main novelty in the statement of Theorem 2 lies in the estimates (14) and (15). These estimates are, in general, best possible.

We shall show first that estimate (14) is best possible in the following sense. Let $\theta$ and $\varepsilon$ be positive real numbers, $r$ an integer with $r \geq 2$, and $p$ a prime number larger than $r$. Then there exist positive integers $k$ and $l$ with $(\theta-\varepsilon) l \leq k \leq(\theta+\varepsilon) l$ and there exists a polynomial $f$ of degree $r$ and discriminant $D$ with $l=\operatorname{ord}_{p} D$ and for which the solutions of (11) are given by $t$ congruences (12) with

$$
\max _{1 \leq i \leq t}\left\{u_{i}(k)\right\}=T .
$$

Note that since $p>r$ and $t \leq r$ the congruences (12) are uniquely determined.

Let $r, t, m$, and $n$ be integers with $r \geq t \geq 2, m>0$, and $m \geq n \geq 0$, and let $p$ be a prime number with $p>r$. We define $f(x)$ by

$$
\begin{aligned}
f(x)= & \left(x+p^{m}\right)\left(x+2 p^{m}\right) \cdots\left(x+t p^{m}\right) \\
& \cdot\left(x+(t+1) p^{n}\right)(x+t+2) \cdots(x+r) .
\end{aligned}
$$

Let $D$ denote the discriminant of $f$. Then $l=\operatorname{ord}_{p} D=t(t-1) m+2 t n$.

Let $s$ be a positive integer with $s \leq m$ and take $t=r$ and $n=0$ in (36). Then $l=r(r-1) m$. Put $k=r s$. The complete solution of $f(x) \equiv 0\left(\bmod p^{k}\right)$ 
is given by $x \equiv 0\left(\bmod p^{s}\right)$. In this case $u_{1}=k-s=((r-1) / r) k$. Since $1 \leq s \leq m, r m=l /(r-1)$, and $m$ is at our disposal, we see that the upper bound for $u_{i}(k)$ in $(14)$ of $T=[((r-1) / r) k]$ is best possible for the range $1 \leq$ $k \leq l /(r-1)$. Next let $j$ be an integer with $1 \leq j \leq r-2$ and take $t=j+1$ in (36). Then $l=j(j+1) m+2(j+1) n$. Put $k=(j+1) m+n$ and $v=k-(j+1) n$. The complete solution of $f(x) \equiv 0\left(\bmod p^{k}\right)$ is given by $x \equiv 0\left(\bmod p^{m}\right)$, or $x \equiv-(j+2) p^{n}\left(\bmod p^{v}\right)$, or $x \equiv-i\left(\bmod p^{k}\right)$ for $i=j+3, \ldots, r$. In this case $u_{1}=k-m=j m+n$, hence $u_{1}=l /((j+1)(j+2))+(j /(j+2)) k$. Note that as $n$ varies from 0 to $m, k$ varies from $l / j$ to $l /(j+1)$. Thus the upper bound for $u_{i}(k)$ in (14) is best possible for $k$ with $l / j \geq k \geq l /(j+1)$ for $j=1, \ldots, r-2$. Finally, take $t=2$ and $n=0$ in (36). Let $s$ be an integer larger than $m$ and put $k=s+m=l+(s-m)$. Then the complete solution of $f(x) \equiv 0\left(\bmod p^{k}\right)$ is given by $x \equiv-p^{m}\left(\bmod p^{s}\right)$, or $x \equiv-2 p^{m}\left(\bmod p^{s}\right)$, or $x \equiv-i\left(\bmod p^{k}\right)$ for $i=3, \ldots, r$, and so $u_{1}=u_{2}=m=l / 2$, whence the upper bound for $u_{i}(k)$ in (14) is best possible for the range $k \geq l$ and therefore for the range $k \geq 1$. Further we note that

$$
u_{1}+u_{2}=l
$$

and that the number of solutions modulo $p^{k}$ of $f(x) \equiv 0\left(\bmod p^{k}\right)$ is $2 p^{m}+r-2$ or equivalently

$$
2 p^{l / 2}+r-2 \text {. }
$$

We shall now show that estimate (15) is best possible for $k \geq l /(r-1)$. For the range $k \geq l$ it suffices to recall (37). Next, as before, let $j$ be an integer with $1 \leq j \leq r-2$ and take $t=j+1$ in (36) so that $l=j(j+1) m+2(j+1) n$. Put $k=(j+1) m+n+1$ and $v=k-(j+1) n$. The complete solution of $f(x) \equiv 0\left(\bmod p^{k}\right)$ is given by $x \equiv-i p^{m}\left(\bmod p^{m+1}\right)$ for $i=1, \ldots, j+1$, or $x \equiv-(j+2) p^{n}\left(\bmod p^{v}\right)$, or $x \equiv-i\left(\bmod p^{k}\right)$ for $i=j+3, \ldots, r$. In this case

$$
u_{1}+\cdots+u_{r}=j(j+1) m+2(j+1) n=l,
$$

and if $n$ is positive then $l / j>k>l /(j+1)$. Further for $n$ positive the number of solutions modulo $p^{k}$ of $f(x) \equiv 0\left(\bmod p^{k}\right)$ is

$$
\begin{aligned}
& (j+1) p^{j m+n}+p^{(j+1) n}+r-j-2 \\
& \quad=(j+1) p^{T}+p^{l-(j+1) T}+r-j-2 .
\end{aligned}
$$

Since $m$ and $n$ are still free to be chosen we see that estimate (15) is best possible for $l / j \geq k \geq l /(j+1)$ for $j=1, \ldots, r-2$ and so for the range $k \geq l /(r-1)$. For $k$ satisfying $1 \leq k<l /(r-1)$ the minimum of $2[l / 2]$ and $r[((r-1) / r) k]$ is $r[((r-1) / r) k]$. In this case estimate $(15)$ is close to optimal as the following example shows. Let $r, w$, and $m$ be positive integers with $w \geq m+1$ and $r \geq 2$. Let $p$ be a prime number with $p>r$. We define $g(x)$ by

$$
g(x)=\left(x+p^{w}\right)\left(x+2 p^{w}\right)\left(x+3 p^{m}\right) \cdots\left(x+r p^{m}\right) .
$$


Let $D$ denote the discriminant of $g$. Then $l=\operatorname{ord}_{p} D=2(w-m)+r(r-1) m$. Put $k=1+r m$. The complete solution of $g(x) \equiv 0\left(\bmod p^{k}\right)$ is given by $x \equiv 0\left(\bmod p^{m+1}\right)$ or $x \equiv-i p^{m}\left(\bmod p^{m+1}\right)$ for $i=3, \ldots, r$. Thus $t=r-1$,

$$
u_{1}+\cdots+u_{t}=(r-1)^{2} m=(r-1)\left[\left(\frac{r-1}{r}\right) k\right] \text {, }
$$

and the number of solutions modulo $p^{k}$ of $g(x) \equiv 0\left(\bmod p^{k}\right)$ is

$$
(r-1) p^{[((r-1) / r) k]}
$$

For any prime $p$ and nonzero integers $r, k$, and $D$ with $r \geq 2$ and $k>0$ we define $Q=Q(r, k, p, D)$ and $B=B(r, k, p, D)$ in the following way. We put $(Q, B)=(r, 0)$ except when $T \neq 0$ and $2[l / 2] / T \leq r$, in which case we put $(Q, B)=\left(Q_{1}, B_{1}\right)$ where

$$
2\left[\frac{l}{2}\right]=Q_{1} T+B_{1}
$$

with $0 \leq Q_{1}$ and $0 \leq B_{1}<T$. Now observe that the single congruence $x \equiv b_{i}\left(\bmod p^{k-u_{i}}\right)$ is equivalent to the $p^{u_{i}}$ congruences $x \equiv a_{j}\left(\bmod p^{k}\right)$, where $a_{j}=b_{i}+j p^{k-u_{i}}$ for $j=1, \ldots, p^{u_{i}}$. Thus the number of solutions modulo $p^{k}$ of $(11)$ is $p^{u_{1}}+\cdots+p^{u_{t}}$. Since for any positive integers $u, v$ with $u \geq v$ we have

$$
p^{u+1}+p^{v-1}>p^{u}+p^{v}
$$

it follows from (14) and (15) that

$$
p^{u_{1}}+\cdots+p^{u_{t}} \leq Q p^{T}+p^{B}+r-Q-1 .
$$

Since $T \leq[l / 2]$ we see that

$$
Q p^{T}+p^{B}+r-Q-1 \leq 2 p^{[l / 2]}+r-2 .
$$

Therefore we have proved the following result.

Corollary 2. Let $p$ be a prime number, $k$ a positive integer, and $f$ a polynomial with integer coefficients, content coprime with $p$, degree $r(\geq 2)$, and nonzero discriminant $D$. The number of solutions modulo $p^{k}$ of

$$
f(x) \equiv 0 \quad\left(\bmod p^{k}\right)
$$

is at most

$$
Q p^{T}+p^{B}+r-Q-1
$$

which in particular is at most

$$
2 p^{[l / 2]}+r-2 \text {. }
$$

In 1921 Nagell [30] and Ore [32] proved independently that the number of solutions modulo $p^{k}$ of $(41)$ is at most $r p^{2 l}$. This was improved by Sándor 
[33] in 1952 to $r p^{l / 2}$ for $k>l$ and in 1981 Huxley [19] obtained the same bound for all positive integers $k$. Estimates (42) and (43) coincide when $k \geq l$ and, by (38), they are best possible for this range.

If $k<l$ then we may have $T<[l / 2]$ in which case $Q p^{T}+p^{B}+r-Q-1$ is smaller than $2 p^{[l / 2]}+r-2$. It follows from (39) that (42) is best possible for the range $l / j \geq k \geq l /(j+1)$ for $j=1, \ldots, r-2$. Finally since $T \leq[((r-1) / r) k]$ for all positive integers $k$ we have

$$
Q p^{T}+p^{B}+r-Q-1 \leq r p^{[((r-1) / r) k]} .
$$

Thus, by virtue of (40), estimate (42) is close to best possible for the range $1 \leq k \leq l /(r-1)$.

By Theorem 2 and the Chinese Remainder Theorem we obtain the following result.

Corollary 3. Let $m$ be a positive integer, and let $f$ be a polynomial with integer coefficients, content coprime with $m$, degree $r(\geq 2)$, and nonzero discriminant $D$. There is an integer $t$ with $0 \leq t \leq r^{\omega(m)}$, nonnegative integers $b_{1}, \ldots, b_{t}$, and positive integers $d_{1}, \ldots, d_{t}$ satisfying

$$
\operatorname{ord}_{p} d_{i} \leq T\left(r, \operatorname{ord}_{p} m, p, D\right)
$$

for $i=1, \ldots, t$ and all prime numbers $p$, such that the complete solution of

$$
f(x) \equiv 0 \quad(\bmod m)
$$

is given by the $t$ mutually disjoint congruences $x \equiv b_{i}\left(\bmod m / d_{i}\right)$ for $i=$ $1, \ldots, t$.

By Corollary 2 and the Chinese Remainder Theorem the number of solutions modulo $m$ of (45) is at most the product over all primes $p$ dividing $m$ of the upper bound given by (42) with $k=\operatorname{ord}_{p} m$. In particular, by (43) and (44), we see that the number of solutions modulo $m$ of (45) is at most

$$
\prod_{p \mid m} \min \left(2 p^{\left[\operatorname{ord}_{p} D / 2\right]}+r-2, r p^{\left[((r-1) / r) \operatorname{ord}_{p} m\right]}\right) .
$$

We remark that the upper bound in (46) is again sharp. Let $w, r$, and $j_{1}, \ldots, j_{w}$ be positive integers with $r \geq 2$, and let $p_{1}, \ldots, p_{w}$ be prime numbers larger than $r$. Put

$$
h(x)=x\left(x+p_{1}^{j_{1}} \cdots p_{w}^{j_{w}}\right)(x+1) \cdots(x+r-2),
$$

let $v$ be an integer with $v \geq w$, and let $p_{w+1}, \ldots, p_{v}$ be primes that are larger than $p_{1}^{j_{1}} \cdots p_{w}^{j_{w}}$. Finally let $k_{1}, \ldots, k_{v}$ be positive integers with $k_{i}>2 j_{i}$ for $i=1, \ldots, w$ and put $m=p_{1}^{k_{1}} \cdots p_{v}^{k_{v}}$. Then by the Chinese Remainder Theorem and the discussion preceding (38), it follows that the number of solutions of (45) with $h$ as in (47) is exactly

$$
\prod_{p \mid m}\left(2 p^{\left.\operatorname{ord}_{p} D / 2\right]}+r-2\right)
$$




\section{Preliminary lemmas}

Let $\alpha$ be an algebraic number of degree $n$ and define the height of $\alpha$, denoted by $h(\alpha)$, by

$$
h(\alpha)=(M(f))^{1 / n},
$$

where $f$ is the minimal polynomial of $\alpha$ over the integers. Let $t$ and $\tau$ be positive numbers such that $t<\sqrt{2 / n}$ and $\sqrt{2-n t^{2}}<\tau<t$, and put $\lambda=2 /(t-\tau)$ and

$$
A_{1}=\frac{t^{2}}{2-n t^{2}}\left(n \log (h(\alpha))+\frac{n}{2}\right) \text {. }
$$

Suppose that $\lambda<n$. A rational number $x / y$ is said to be a very good approximation to $\alpha$ if

$$
|\alpha-x / y|<\left(4 e^{A_{1}} H(x, y)\right)^{-\lambda},
$$

where $H(x, y)=\max (|x|,|y|)$. Bombieri and Schmidt [5], building on the earlier work of Bombieri [2] and Bombieri and Mueller [4], and of course the classical work of Thue and Siegel, proved the following result.

Thue-Siegel principle. If $\alpha$ is of degree $n(\geq 3)$ and $x / y$ and $x^{\prime} / y^{\prime}$ are two very good approximations to $\alpha$ then

$$
\log \left(4 e^{A_{1}}\right)+\log \left(H\left(x^{\prime}, y^{\prime}\right)\right) \leq \gamma^{-1}\left(\log \left(4 e^{A_{1}}\right)+\log (H(x, y))\right),
$$

where $\gamma=\left(n t^{2}+\tau^{2}-2\right) /(n-1)$.

We must also deal with the possibility that $\alpha$ is of degree 1 or 2 . In this case we appeal to the following simple result.

Lemma 1. Let $\alpha$ be an algebraic number with minimal polynomial $f$ over $\mathbb{Q}$, and let $a$ be the leading coefficient of $f$ and $D$ the discriminant of $f$. Suppose that $p / q$ is a rational number with $q \neq 0$. If $\alpha$ is a rational and $\alpha \neq p / q$ then

$$
\left|\alpha-\frac{p}{q}\right| \geq \frac{1}{|a q|} \geq \frac{1}{M(f)|q|} .
$$

If $\alpha$ is of degree 2 over $\mathbb{Q}$ then

$$
\left|\alpha-\frac{p}{q}\right| \geq \min \left(M(f)^{-1},\left(2|D|^{1 / 2} q^{2}\right)^{-1}\right) .
$$

Proof. First assume that $\alpha$ is of degree 1 . Then $f(x)=a x-b$ with $a$ and $b$ coprime integers and with $a \neq 0$. Thus $\alpha=b / a$ and for any rational number $p / q$ with $q \neq 0$ and $\alpha \neq p / q$,

$$
\left|\alpha-\frac{p}{q}\right|=\left|\frac{b}{a}-\frac{p}{q}\right| \geq \frac{1}{|a q|} .
$$

Since $M(f) \geq|a|$ the result holds.

Next assume that $\alpha$ is of degree 2. Then $f(x)=a x^{2}+b x+c$ with $a \neq 0$. Let $\alpha^{\prime}$ denote the other root of $f$. For any rational number $p / q$ with $q \neq 0$,

$$
\left|\alpha-\frac{p}{q}\right|=\frac{\left|a p^{2}+b p q+c q^{2}\right|}{q^{2}|a|\left|\alpha^{\prime}-p / q\right|} \geq \frac{1}{q^{2}|a|\left|\alpha^{\prime}-p / q\right|} .
$$


Now either

$$
\left|\alpha-\frac{p}{q}\right| \geq\left|\alpha-\alpha^{\prime}\right|=\frac{|D|^{1 / 2}}{|a|} \geq \frac{1}{|a|} \geq \frac{1}{M(f)},
$$

or

In the latter case, by (48),

$$
\left|\alpha^{\prime}-\frac{p}{q}\right|=\left|\left(\alpha^{\prime}-\alpha\right)+\left(\alpha-\frac{p}{q}\right)\right| \leq 2\left|\alpha^{\prime}-\alpha\right| \leq 2 \frac{|D|^{1 / 2}}{|a|} \text {. }
$$

$$
\left|\alpha-\frac{p}{q}\right| \geq \frac{1}{2|D|^{1 / 2} q^{2}}
$$

and so the result follows from (49) and (50).

From the proof of Lemma 1 of Lewis and Mahler [21] together with refinements (a) and (b) of Bombieri and Schmidt [5, p. 72] we obtain the following result.

Lemma 2. Let $f$ be a polynomial with coefficients from the complex numbers $\mathbb{C}$, degree $n(\geq 2)$, and zeros $\alpha_{1}, \ldots, \alpha_{n}$ in $\mathbb{C}$. For every $z$ in $\mathbb{C}$,

$$
|f(z)| \geq \frac{|D(f)|^{1 / 2}}{n^{(n-1) / 2} 2^{n-1} M(f)^{n-2}} \min _{1 \leq i \leq n}\left|z-\alpha_{i}\right| .
$$

We may apply Lemma 2 to obtain the following version of Lemma 1 of [5].

Lemma 3. Let $F$ be a binary form of degree $r(\geq 3)$ with integer coefficients and nonzero discriminant $D(F)$. For every pair of integers $(x, y)$ with $y \neq 0$

$$
\min _{\alpha}\left|\alpha-\frac{x}{y}\right| \leq \frac{2^{r-1} r^{(r-1) / 2}(M(F))^{r-2}|F(x, y)|}{|D(F)|^{1 / 2}|y|^{r}},
$$

where the minimum is taken over the zeros $\alpha$ of $F(z, 1)$.

Proof. Put $f(z)=F(z, 1)$ and denote the degree of $f$ by $n$. Since $F$ has degree at least 3 and $D(F) \neq 0, n$ is at least 2 and so by Lemma 2

$$
|F(x, y)||y|^{-r}=\left|f\left(\frac{x}{y}\right)\right| \geq \frac{|D(f)|^{1 / 2}}{n^{(n-1) / 2} 2^{n-1}(M(f))^{n-2}} \min _{\alpha}\left|\alpha-\frac{x}{y}\right| .
$$

Since $F$ is homogeneous, $M(f)=M(F)$. Let

$$
F(x, y)=a_{r} x^{r}+a_{r-1} x^{r-1} y+\cdots+a_{0} y^{r} .
$$

If $a_{r} \neq 0$ then $n=r, D(F)=D(f)$, and the result follows immediately. If $a_{r}=0$ then $a_{r-1} \neq 0, n=r-1$, and $|D(F)|^{1 / 2}=\left|a_{r-1}\right||D(f)|^{1 / 2}$. But then $M(f) \geq\left|a_{r-1}\right|$ and the result again follows.

\section{Proof OF THEOREM 1}

Let $p$ be a prime and suppose that $p^{k}$ exactly divides $h$. If $(x, y)$ is a primitive solution of (1) then certainly

$$
F(x, y) \equiv 0 \quad\left(\bmod p^{k}\right) .
$$


If $p$ does not divide $y$ then $y$ is invertible modulo $p^{k}$ and so

$$
F\left(x y^{-1}, 1\right) \equiv 0 \quad\left(\bmod p^{k}\right) .
$$

By Theorem 2 there is an integer $t$ with $0 \leq t \leq s$, where $s$ denotes the number of zeros of $F(z, 1)$ in $R_{p}$, and there are integers $b_{1}, \ldots, b_{t}$ and $u_{1}, \ldots, u_{t}$ with $u_{i}$ satisfying $(14)$ such that $x y^{-1} \equiv b_{i}\left(\bmod p^{k-u_{i}}\right)$ for some integer $i$ with $1 \leq i \leq t$. We suppose, as we may without loss of generality, that for each integer $j$ with $1 \leq j \leq t$ there is a primitive solution $(x, y)$ of $(1)$ for which $x y^{-1} \equiv b_{j}\left(\bmod p^{k-u_{j}}\right)$. Note also that since $D(F(z, 1))$ divides $D(F)$ $(=D(F(X, Y)))$ we may take $D(F)$ in place of $D(F(z, 1))$ in estimate (14).

Put $F_{i}(X, Y)=F\left(p^{k-u_{i}} X+b_{i} Y, Y\right)$ for $i=1, \ldots, t$. By Theorem 2 the content of $F_{i}$ is divisible by $p^{k}$. Since $F$ has content 1 the content of $F_{i}$ is a power of $p$ and since $p^{k}$ exactly divides $h$ and there is a primitive solution $(x, y)$ of $(1)$ for which $x y^{-1} \equiv b_{i}\left(\bmod p^{k-u_{i}}\right)$ the content of $F_{i}$ is $p^{k}$ for $i=1, \ldots, t$. Put $\widetilde{F}_{i}(X, Y)=p^{-k} F_{i}(X, Y)$ for $i=1, \ldots, t$. Plainly $\widetilde{F}_{i}$ has content 1 and by (5) and (6)

$$
D\left(\widetilde{F}_{i}\right)=\frac{p^{\left(k-u_{i}\right) r(r-1)} D(F)}{p^{k 2(r-1)}}
$$

for $i=1, \ldots, t$. Further since $x y^{-1} \equiv b_{i}\left(\bmod p^{k-u_{i}}\right)$, there exists an integer $x_{i}$ for which $x=p^{k-u_{i}} x_{i}+b_{i} y$. Thus $\left(x_{i}, y\right)$ is a primitive solution of $\widetilde{F}_{i}(X, Y)=h p^{-k}$.

Similarly if $(x, y)$ is a primitive solution of (1) and $p$ divides $y$ then $p$ does not divide $x$ and so $x$ is invertible modulo $p^{k}$. In this case $F\left(1, y x^{-1}\right) \equiv$ $0\left(\bmod p^{k}\right)$. By Theorem 2 applied to $F(1, z)$ there is an integer $w$ with $t \leq$ $w$ and there are integers $b_{t+1}, \ldots, b_{w}$ and $u_{t+1}, \ldots, u_{w}$, with $u_{i}$ satisfying (14) with $D(F)$ in place of $D(F(1, z))$, such that $y x^{-1} \equiv b_{i}\left(\bmod p^{k-u_{i}}\right)$ for some integer $i$ with $t+1 \leq i \leq w$. We choose $w$ to be minimal. Since $p$ divides $y$ it also divides $b_{i}$ for $i=t+1, \ldots, w$ and thus by Theorem $2, w-t$ is at most $s_{1}$, where $s_{1}$ is the number of roots $\alpha$ of $F(1, z)$ with $|\alpha|_{p}<1$. Since each nonzero root of $F(1, z)$ is the inverse of a nonzero root of $F(z, 1), w \leq r$. Arguing as before, but with the roles of $x$ and $y$ reversed, we determine binary forms $\widetilde{F}_{i}$ of content 1 that satisfy (51) for $i=t+1, \ldots, w$.

Therefore if $(x, y)$ is a primitive solution of (1) then it determines a triple $\left(i, x^{\prime}, y^{\prime}\right)$, where $1 \leq i \leq w$ and $\left(x^{\prime}, y^{\prime}\right)$ is a pair of coprime integers for which $\widetilde{F}_{i}\left(x^{\prime}, y^{\prime}\right)=h p^{-k}$. Further, distinct primitive solutions of (1) determine distinct triples. We may assume, without loss of generality, that $\operatorname{ord}_{p} g=\operatorname{ord}_{p} h$ for all primes $p$ that divide $g$. Then, by repeating the above construction for each prime $p$ that divides $g$ we obtain a set $W$ of at most $r^{\omega(g)}$ binary forms with the property that distinct primitive solutions $(x, y)$ of $(1)$ correspond to distinct triples $\left(\widehat{F}, x^{\prime}, y^{\prime}\right)$, where $\widehat{F}$ is in $W$ and $\left(x^{\prime}, y^{\prime}\right)$ is a pair of coprime 
integers for which $\widehat{F}\left(x^{\prime}, y^{\prime}\right)=h / g$. Further if $\widehat{F}$ is in $W$ then $\widehat{F}$ has content 1 and, by (51) and Theorem 2 ,

$$
|D(\widehat{F})| \geq \frac{g^{(r-2)(r-1)}|D(F)|}{G(g, r, D)^{r(r-1)}},
$$

hence, by (8),

$$
|D(\widehat{F})| \geq\left(\left|\frac{h}{g}\right|^{2 / r+\varepsilon}\right)^{r(r-1)} .
$$

Let $\widehat{F}$ be a form in $W$, let $p$ a prime number, and let

$$
B_{0}=\left(\begin{array}{ll}
p & 0 \\
0 & 1
\end{array}\right), \quad B_{j}=\left(\begin{array}{cc}
0 & -1 \\
p & j
\end{array}\right)
$$

for $j=1, \ldots, p$. Then, as in [5], we have

$$
\mathbb{Z}^{2}=\bigcup_{j=0}^{p} B_{j} \mathbb{Z}^{2}
$$

and the number of primitive solutions of $\widehat{F}(x, y)=h / g$ is at most $n_{0}+n_{1}+$ $\cdots+n_{p}$, where $n_{j}$ is the number of primitive solutions of

$$
\widehat{F}_{B_{j}}(x, y)=h / g \text {. }
$$

By (6),

$$
\left|D\left(\widehat{F}_{B_{j}}\right)\right|=p^{r(r-1)}|D(\widehat{F})| \text {. }
$$

Put $n=h / g$ and take $p=41$ in (53). Then, by (52) and (53), the number of primitive solutions of $(1)$ is at most $42 r^{\omega(g)}$ times the maximum number of primitive solutions $(x, y)$ of

$$
G(x, y)=n
$$

for all binary forms $G$ of degree $r$ and for which

$$
|D(G)| \geq\left(41|n|^{2 / r+\varepsilon}\right)^{r(r-1)} \text {. }
$$

Suppose that $G$ is such a form and that $\left(x_{0}, y_{0}\right)$ is a primitive solution of (54). Then there is an $A$ in $\operatorname{GL}(2, \mathbb{Z})$ for which $A^{-1}\left(x_{0}, y_{0}\right)$ is $(1,0)$ and so $(1,0)$ is a solution of $G_{A}(x, y)=n$. Note that $G_{A}$ has leading coefficient $n$. Thus we may suppose that $G$ has leading coefficient $n$ and that $M(G)$ is smallest among all equivalent forms that have $n$ as their leading coefficient.

Let $Y_{0}$ be a positive real number. We shall now estimate the primitive solutions $(x, y)$ of $(54)$ for which $0<y \leq Y_{0}$, and here we shall repeat the argument of Bombieri and Schmidt with some minor changes. We have

$$
G(x, y)=n\left(x-\alpha_{1} y\right) \cdots\left(x-\alpha_{r} y\right),
$$

where $\alpha_{1}, \ldots, \alpha_{r}$ are distinct complex numbers. Put $L_{i}(x, y)=x-\alpha_{i} y$ for $i=1, \ldots, r$. Then by the same argument given for the proof of Lemma 3 of [5] we obtain the next result. 
Lemma 4. Suppose $(x, y)$ and $\left(x_{0}, y_{0}\right)$ are primitive solutions of (54). Then for $1 \leq i, j \leq r$,

$$
\frac{L_{i}\left(x_{0}, y_{0}\right)}{L_{i}(x, y)}-\frac{L_{j}\left(x_{0}, y_{0}\right)}{L_{j}(x, y)}=\left(\beta_{i}-\beta_{j}\right)\left(x y_{0}-x_{0} y\right),
$$

where $\beta_{1}, \ldots, \beta_{r}$ depend on $(x, y)$ and are such that the form

$$
J(u, w)=n\left(u-\beta_{1} w\right) \cdots\left(u-\beta_{r} w\right)
$$

is equivalent to $G$.

We may take $\left(x_{0}, y_{0}\right)=(1,0)$ in which case, by $(56)$,

$$
\frac{1}{L_{i}(x, y)}-\frac{1}{L_{j}(x, y)}=\left(\beta_{j}-\beta_{i}\right) y .
$$

For every primitive solution $(x, y)$ of $(54)$ we choose $j=j(x, y)$ with $\left|L_{j}(x, y)\right| \geq 1$. Then

$$
\frac{1}{\left|L_{i}(x, y)\right|} \geq\left|\beta_{j}-\beta_{i}\right||y|-1 .
$$

Since $\left|\overline{L_{j}(x, y)}\right| \geq 1,(57)$ holds with $\overline{\beta_{j}}$ in place of $\beta_{j}$ and so

$$
\frac{1}{\left|L_{i}(x, y)\right|} \geq\left|\operatorname{Re}\left(\beta_{j}\right)-\beta_{i}\right||y|-1 .
$$

We now choose an integer $m=m(x, y)$ with $\left|m-\operatorname{Re}\left(\beta_{j}\right)\right| \leq 1 / 2$ and we obtain

$$
\frac{1}{\left|L_{i}(x, y)\right|} \geq\left(\left|m-\beta_{i}\right|-\frac{1}{2}\right)|y|-1
$$

for $i=1, \ldots, r$.

For $1 \leq i \leq r$, let $X_{i}$ be the set of primitive solutions of (54) with $1 \leq y \leq Y_{0}$ and $\left|L_{i}(x, y)\right| \leq 1 / 2 y$.

Lemma 5. Suppose $(x, y) \neq\left(x^{\prime}, y^{\prime}\right)$ are in $X_{i}$ with $y \leq y^{\prime}$. Then

$$
\frac{y^{\prime}}{y} \geq \frac{2}{7} \max \left(1,\left|\beta_{i}-m\right|\right),
$$

where $\beta_{i}=\beta_{i}(x, y)$ and $m=m(x, y)$.

Proof. This is Lemma 4 of [5] and the proof goes through unchanged.

Similarly we obtain the following version of Lemma 5 of [5].

Lemma 6. Suppose $(x, y)$ is a primitive solution of (54) with $y>0$ and $\left|L_{i}(x, y)\right|>1 / 2 y$. Then

$$
\left|m-\beta_{i}\right| \leq \frac{7}{2},
$$

where again $\beta_{i}=\beta_{i}(x, y)$ and $m=m(x, y)$.

For each set $X_{i}$ that is not empty let $\left(x^{(i)}, y^{(i)}\right)$ be the element with the largest value of $y$. Let $X$ be the set of solutions of (54) with $1 \leq y \leq Y_{0}$ minus the elements $\left(x^{(1)}, y^{(1)}\right), \ldots,\left(x^{(r)}, y^{(r)}\right)$. 
Let $i$ be an integer with $1 \leq i \leq r$ and, when $X_{i}$ is nonempty, let $\left(x_{1}^{(i)}, y_{1}^{(i)}\right)$, $\ldots,\left(x_{\nu}^{(i)}, y_{\nu}^{(i)}\right)$ be the elements of $X_{i}$ with $y_{1}^{(i)} \leq \cdots \leq y_{\nu}^{(i)}$. Thus $\left(x_{\nu}^{(i)}, y_{\nu}^{(i)}\right)=$ $\left(x^{(i)}, y^{(i)}\right)$. By Lemma 5

$$
\frac{2}{7} \max \left(1,\left|\beta_{i}\left(x_{k}^{(i)}, y_{k}^{(i)}\right)-m\left(x_{k}^{(i)}, y_{k}^{(i)}\right)\right|\right) \leq \frac{y_{k+1}^{(i)}}{y_{k}^{(i)}}
$$

for $k=1, \ldots, \nu-1$, hence

$$
\prod_{(x, y) \in X \cap X_{i}}\left(\frac{2}{7} \max \left(1,\left|\beta_{i}(x, y)-m(x, y)\right|\right)\right) \leq Y_{0} .
$$

For $(x, y)$ in $X$ but not in $X_{i}$ we have

$$
\frac{2}{7} \max \left(1,\left|\beta_{i}(x, y)-m(x, y)\right|\right) \leq 1
$$

by Lemma 6 . Thus

$$
\prod_{(x, y) \in X}\left(\frac{2}{7} \max \left(1,\left|\beta_{i}(x, y)-m(x, y)\right|\right)\right) \leq Y_{0} .
$$

By Lemma 4 the form

$$
J(u, w)=n \prod_{i=1}^{r}\left(u-\beta_{i} w\right)
$$

is equivalent to $G$ and thus so also is the form

$$
\widehat{J}(u, w)=n \prod_{i=1}^{r}\left(u-\left(\beta_{i}-m\right) w\right) .
$$

Therefore

$$
\prod_{i=1}^{r} \max \left(1,\left|\beta_{i}(x, y)-m(x, y)\right|\right)=\frac{M(\widehat{J})}{|n|} \geq \frac{M(G)}{|n|} .
$$

Taking the product of (59) for $i=1, \ldots, r$ we find that

$$
\left(\left(\frac{2}{7}\right)^{r} \frac{M(G)}{|n|}\right)^{|X|} \leq Y_{0}^{r}
$$

here $|X|$ denotes the cardinality of $X$. By a result of Mahler [25],

$$
M(G) \geq\left(\frac{|D(G)|}{r^{r}}\right)^{1 /(2 r-2)},
$$

and thus, by (55),

$$
M(G) \geq \frac{\left(41|n|^{2 / r+\varepsilon}\right)^{r / 2}}{r^{r /(2 r-2)}} .
$$

Since $r^{1 /(2 r-2)} \leq 3^{1 / 4}$ we find that

$$
M(G) \geq\left(\frac{41^{1 / 2}}{3^{1 / 4}}\right)^{r}|n|^{1+\varepsilon r / 2} .
$$


For any positive real numbers $a, b, c, d$ we have $(a+b) /(c+d) \leq$ $\max (a / c, b / d)$ and thus

$$
\begin{gathered}
\frac{\log |n|+r \log (7 / 2)}{(1+(\varepsilon r / 2)) \log |n|+r \log \left(41^{1 / 2} / 3^{1 / 4}\right)} \\
\leq \max \left(\frac{2}{2+\varepsilon r}, \frac{\log (7 / 2)}{\log \left(41^{1 / 2} / 3^{1 / 4}\right)}\right) .
\end{gathered}
$$

Therefore, by (61) and (62),

$$
\left(\frac{2}{7}\right)^{r} \frac{M(G)}{|n|} \geq M(G)^{\theta},
$$

where $\theta=\min \left(\varepsilon r /(2+\varepsilon r), \theta_{1}\right)$ and $\theta_{1}=1-(\log (7 / 2)) /\left(\log \left(41^{1 / 2} / 3^{1 / 4}\right)\right)$. Accordingly, by (60) and (63),

$$
|X| \leq \frac{r \log Y_{0}}{\theta \log M(G)} .
$$

We now take $Y_{0}=M(G)^{2}$ so that $|X| \leq 2 r / \theta$. Thus the number of primitive solutions $(x, y)$ of (54) with $1 \leq y \leq M(G)^{2}$ is at most $(2 r / \theta)+r$ and therefore, since $|D(G)|=|D(-G)|$ and $M(G)=M(-G)$, the number with $|y| \leq M(G)^{2}$ is at most $2((2 r / \theta)+r+1)$.

We shall now estimate the number of primitive solutions $(x, y)$ of (54) with $|y| \geq M(G)^{2}$. To each such primitive solution $(x, y)$ we associate a root $\alpha_{i}$ of $G(x, 1)$ for which

$$
\left|\alpha_{i}-\frac{x}{y}\right| \leq\left|\alpha_{j}-\frac{x}{y}\right|
$$

for $j=1, \ldots, r$. For $i=1, \ldots, r$ let $I^{(i)}$ denote the set of such solutions associated to $\alpha_{i}$.

Now fix $i$ and let $\left(x_{1}, y_{1}\right),\left(x_{2}, y_{2}\right), \ldots$ denote the elements of $I^{(i)}$ with $y_{j}>0$ for $j=1,2, \ldots$, ordered so that $y_{1} \leq y_{2} \leq \ldots$. By Lemma 3 ,

$$
\left|\alpha_{i}-\frac{x_{j}}{y_{j}}\right| \leq \frac{2^{r-1} r^{(r-1) / 2} M(G)^{r-2}|n|}{|D(G)|^{1 / 2} y_{j}^{r}}
$$

for $j=1,2, \ldots$, and so

$$
\left|\frac{x_{j+1}}{y_{j+1}}-\frac{x_{j}}{y_{j}}\right| \leq \frac{2^{r} r^{(r-1) / 2}|n|}{|D(G)|^{1 / 2}} \frac{M(G)^{r-2}}{y_{j}^{r}} .
$$

Since $\left|x_{j+1} y_{j}-x_{j} y_{j+1}\right| \geq 1$, we have, by (55),

$$
\frac{y_{j}^{r-1}}{M(G)^{r-2}} \leq y_{j+1} \text {. }
$$

We define the positive real number $\delta_{j}$ for each integer $j$ for which there exists an element $\left(x_{j}, y_{j}\right)$ in $I^{(i)}$ by

$$
y_{j}=M(G)^{1+\delta_{j}} .
$$


By $(61) M(G)>1$ and so, by $(65),(r-1) \delta_{j} \leq \delta_{j+1}$ for $j=1,2, \ldots$ Note that $\delta \geq 1$ since $y_{1} \geq M(G)^{2}$. Therefore

$$
(r-1)^{j-1} \leq \delta_{j}
$$

for $j=1,2, \ldots$, and for any positive integers $k$ and $l$ with $\left(x_{k+l}, y_{k+l}\right)$ in $I^{(i)}$,

$$
\delta_{k}(r-1)^{l} \leq \delta_{k+l}
$$

Let $f_{i}$ denote the minimal polynomial of $\alpha_{i}$ over the rationals. Then $f_{i}$ divides $G(x, 1)$ in $\mathbb{Z}[x], M\left(f_{i}\right) \leq M(G)$, and $\left|D\left(f_{i}\right)\right| \leq|D(G)|$. We suppose first that $\alpha_{i}$ is a rational number. Then, by (55), (64), and Lemma 1,

$$
y_{j}^{r-1} \leq M(G)^{r-1}
$$

which is impossible since $y_{1} \geq M(G)^{2}$. Next suppose that $\alpha_{i}$ is of degree 2 over the rationals. Then by (55), (61), (64), and Lemma 1,

$$
y_{j}^{r-2} \leq\left(2^{r} r^{(r-1) / 2}|n|\right) M(G)^{r-2}<M(G)^{2(r-2)},
$$

and again this is impossible since $y_{1} \geq M(G)^{2}$.

Finally suppose that $\alpha_{i}$ is of degree $d$ over the rationals with $d$ at least 3 . We shall apply the Thue-Siegel principle with

$$
t=\sqrt{2 /\left(d+a^{2}\right)}, \quad a=.1
$$

and

$$
\tau=1.2 \sqrt{2-d t^{2}}=1.2 a t=.12 t .
$$

Then $\lambda=2 /(t-\tau)=2 /(.88 t)$ and so $\lambda<.93 d \leq .93 r$. Further, $t^{2} /\left(2-d t^{2}\right)=$ $a^{-2}=100$ so

$$
A_{1}=100\left(d \log \left(h\left(\alpha_{i}\right)\right)+d / 2\right)=100\left(\log \left(M\left(f_{i}\right)\right)+d / 2\right)
$$

and

$$
\gamma=\left(d t^{2}+\tau^{2}-2\right) /(d-1)=\left((.12)^{2}-(.01)\right) 2 /((d+.01)(d-1)),
$$

hence

$$
\gamma^{-1}<172(d-1)^{2} \leq 172(r-1)^{2} .
$$

Now observe that

$$
4 e^{A_{1}} \leq 4 M\left(f_{i}\right)^{100} e^{50 d} \leq 4 M(G)^{100} e^{50 r} .
$$

By $(61), M(G) \geq\left(41^{1 / 2} / 3^{1 / 4}\right)^{r}$ and so

$$
8 e^{50 r} \leq\left(8^{1 / 3} e^{50}\right)^{r}<M(G)^{33} .
$$

Therefore

$$
8 e^{A_{1}}<M(G)^{133}
$$


Note that by (55) and (64), $\left|\alpha_{i}-x_{j} / y_{j}\right|<1$, hence $\left|x_{j}\right|<\left|y_{j}\right|\left(\left|\alpha_{i}\right|+1\right) \leq$ $2 M(G) y_{j}$. Thus $H\left(x_{j}, y_{j}\right)<2 M(G) y_{j}$ and so

$$
\left(4 e^{A_{1}} H\left(x_{j}, y_{j}\right)\right)^{\lambda}<M(G)^{\left(135+\delta_{j}\right) \lambda}<M(G)^{\left(135+\delta_{j}\right)(.93 r)} .
$$

By (55), (64), and (66),

$$
\left|\alpha_{i}-x_{j} / y_{j}\right|<M(G)^{-\delta_{j} r} .
$$

It follows from (71) and (72) that $x_{j} / y_{j}$ is a very good approximation to $\alpha_{i}$ whenever

$$
\delta_{j} r \geq\left(135+\delta_{j}\right)(.93 r),
$$

hence for $\delta_{j} \geq 1794$. But $\delta_{j} \geq(r-1)^{j-1}$ and so if we put

$$
k=1+\left[\frac{\log 1794}{\log (r-1)}\right] \text {, }
$$

then $x_{k+1} / y_{k+1}, x_{k+2} / y_{k+2}, \ldots, x_{k+l} / y_{k+l}$ are all very good approximations to $\alpha_{i}$ whenever $\left(x_{k+l}, y_{k+l}\right)$ is in $I^{(i)}$. Suppose that there exists an integer $l$ with $l \geq 2$ for which $\left(x_{k+l}, y_{k+l}\right)$ is in $I^{(i)}$. Then by the Thue-Siegel principle and (69),

$$
\log \left(4 e^{A_{1}}\right)+\log y_{k+l} \leq 172(r-1)^{2}\left(\log \left(4 e^{A_{1}}\right)+\log \left(2 M(G) y_{k+1}\right)\right),
$$

and so, by (70),

$$
\log y_{k+l} \leq 172(r-1)^{2}\left(134 \log M(G)+\log y_{k+1}\right) .
$$

Thus, recall (66),

$$
\delta_{k+l} \leq 172(r-1)^{2}\left(135+\delta_{k+1}\right) .
$$

Since $\delta_{k+1} \geq 1794$, we find that $\delta_{k+l} / \delta_{k+1} \leq 185(r-1)^{2}$. Thus, by (68), $(r-1)^{l-3} \leq 185$, whence

$$
l \leq 3+\frac{\log 185}{\log (r-1)}
$$

Therefore the number of primitive solutions in $I^{(i)}$ is at most

$$
2(4+\log 331890 / \log (r-1))
$$

for $i=1, \ldots, r$. Consequently the number of primitive solutions of $(1)$ is at most

$$
42 r^{\omega(g)}(2((2 r / \theta)+r+1)+2 r(4+\log 331890 / \log (r-1))) .
$$

This in turn is at most

$$
\begin{aligned}
& 84 r^{1+\omega(g)}\left(\max \left(\frac{2}{\theta_{1}}, 2+\frac{4}{\varepsilon r}\right)+1+\frac{1}{r}+4+\frac{\log 331890}{\log (r-1)}\right) \\
& \leq \max \left(2800,2160+\frac{336}{\varepsilon r}\right) r^{1+\omega(g)} \leq 2800\left(1+\frac{1}{8 \varepsilon r}\right) r^{1+\omega(g)},
\end{aligned}
$$

as required. 


\section{LOWER BOUNDS FOR THE NUMBER OF SOLUTIONS OF THUE EQUATIONS}

Silverman [37], extending earlier work of Mahler [24] and Chowla [8], has shown that there exist cubic binary forms $F$, with nonzero discriminant, for which the number of solutions of the Thue equation $(1)$ exceeds $c(\log |h|)^{2 / 3}$ for infinitely many integers $h$, where $c$ is some positive constant. However, the solutions constructed are generally not primitive solutions and as we remarked with Erdös and Tijdeman [10] it may be that there exists a number $C_{1}(r)$, which depends on $r$ only, such that (1) has at most $C_{1}(r)$ primitive solutions whenever $F$ has nonzero discriminant and degree $r$ at least three. Bombieri and Schmidt [5] showed that we may have at least $r$ distinct primitive solutions of (1). They gave the example

$$
F(x, y)=x^{r}+a(x-y)(2 x-y) \cdots(r x-y),
$$

where $a$ is a nonzero integer. Then $(1,1),(1,2), \ldots,(1, r)$ are primitive solutions of $F(x, y)=1$. We do not believe that for a fixed form $F$ there are infinitely many integers $h$ for which (1) has this many primitive solutions if $r$ is large. Indeed we conjecture that there exists an absolute constant $c_{0}$ such that for any binary form $F \in \mathbb{Z}[x, y]$ with nonzero discriminant and degree at least three there exists a number $C$, which depends on $F$, such that if $h$ is an integer larger than $C$ then the Thue equation (1) has at most $c_{0}$ solutions in coprime integers $x$ and $y$. For each binary form $F$ let $\nu(F)$ denote the largest integer $k$ such that (1) has at least $k$ primitive solutions for arbitrarily large integers $h$; if $k$ does not exist put $\nu(F)=\infty$. Next, for each integer $r$ let $\nu^{*}(r)$ be the supremum of $\nu(F)$ over those binary forms $F$ with integer coefficients, nonzero discriminant, and degree $r$. Of course if the above conjecture is valid then $\nu^{*}(r) \leq c_{0}$ for $r=3,4, \ldots$ In this section we shall prove the following result.

Theorem 3. We have

$$
\nu^{*}(3) \geq 18, \quad \nu^{*}(4) \geq 16, \quad \nu^{*}(5) \geq 6,
$$

and

$$
\begin{array}{lrr}
\nu^{*}(6 k) \geq 12, & \nu^{*}(6 k+1) \geq 2, & \nu^{*}(6 k+2) \geq 12, \\
\nu^{*}(6 k+3) \geq 6, & \nu^{*}(6 k+4) \geq 8, & \nu^{*}(6 k+5) \geq 6
\end{array}
$$

for $k=1,2, \ldots$.

Thus $c_{0}$ is at least 18 . To prove Theorem 3 we shall determine various binary forms that are invariant under subgroups of $\operatorname{GL}(2, \mathbb{Z})$. Further, for (73) we shall also make use of parametric solutions of equations of the form $F(u, v)=F(r, s)$.

Let

$$
A=\left(\begin{array}{ll}
a & b \\
c & d
\end{array}\right)
$$


be in $\mathrm{GL}(2, \mathbb{Z})$. Recall that if $x, y$ is a coprime solution of $F_{A}(x, y)=h$ for some integer $h$, then $(a x+b y, c x+d y)$ is a coprime solution of $F(X, Y)=h$. We remark that if $F$ is a form such that $F_{A}=F$ and $(x, y)$ is a primitive solution of (1) then also $A(x, y)=(a x+b y, c x+d y), A^{2}(x, y), A^{3}(x, y), \ldots$ are primitive solutions and so we obtain many primitive solutions of (1). Plainly we may restrict our attention to those elements $A$ of finite order in $\operatorname{GL}(2, \mathbb{Z})$. In fact we shall look for forms $F$ that are invariant under the action of a finite subgroup of $\mathrm{GL}(2, \mathbb{Z})$. Here again we may restrict our attention, this time to equivalence classes of subgroups of $\mathrm{GL}(2, \mathbb{Z})$ under conjugation. For let $G$ be a finite subgroup of $\mathrm{GL}(2, \mathbb{Z})$, and let $F$ be a binary form that is invariant under $G$, that is, $F_{A}=F$ for all $A$ in $G$. Then, for any element $T$ in $\operatorname{GL}(2, \mathbb{Z}), F_{T}$ is invariant under $T G T^{-1}$. There are in total 13 mutually nonconjugate finite subgroups of $\operatorname{GL}(2, \mathbb{Z})$ and they are given in Table 1 (see p. 179 of [31]).

We shall now determine those homogeneous binary forms of small degrees that are invariant under the above 13 groups.

Plainly every binary form is invariant under $C_{1}$ and every form of even

TABLE 1

\begin{tabular}{l|r||l|l}
\hline Group & Generators & Group & Generators \\
\hline$C_{1}$ & $\left(\begin{array}{ll}1 & 0 \\
0 & 1\end{array}\right)$ & $D_{2}$ & $\left(\begin{array}{rr}1 & 0 \\
0 & -1\end{array}\right),\left(\begin{array}{rr}-1 & 0 \\
0 & -1\end{array}\right)$ \\
$C_{2}$ & $\left(\begin{array}{rr}-1 & 0 \\
0 & -1\end{array}\right)$ & $D_{2}^{*}$ & $\left(\begin{array}{rr}0 & 1 \\
1 & 0\end{array}\right),\left(\begin{array}{rr}-1 & 0 \\
0 & -1\end{array}\right)$ \\
$C_{3}$ & $\left(\begin{array}{rr}0 & 1 \\
-1 & -1\end{array}\right)$ & $D_{3}$ & $\left(\begin{array}{rr}0 & 1 \\
1 & 0\end{array}\right),\left(\begin{array}{rr}0 & 1 \\
-1 & -1\end{array}\right)$ \\
$C_{4}$ & $\left(\begin{array}{rr}0 & 1 \\
-1 & 0\end{array}\right)$ & $D_{3}^{*}$ & $\left(\begin{array}{rr}0 & -1 \\
-1 & 0\end{array}\right),\left(\begin{array}{rr}0 & 1 \\
-1 & -1\end{array}\right)$ \\
$C_{6}$ & $\left(\begin{array}{rr}0 & -1 \\
1 & 1\end{array}\right)$ & $D_{4}$ & $\left(\begin{array}{rr}0 & 1 \\
1 & 0\end{array}\right),\left(\begin{array}{rr}0 & 1 \\
-1 & 0\end{array}\right)$ \\
$D_{1}$ & $\left(\begin{array}{rr}1 & 0 \\
0 & -1\end{array}\right)$ & $D_{6}$ & $\left(\begin{array}{ll}0 & 1 \\
1 & 0\end{array}\right),\left(\begin{array}{rr}0 & 1 \\
-1 & 1\end{array}\right)$ \\
$D_{1}^{*}$ & $\left(\begin{array}{rr}0 & 1 \\
1 & 0\end{array}\right)$ & & \\
\hline
\end{tabular}


degree is invariant under $C_{2}$. Forms $F$ invariant under $C_{3}$ satisfy

$$
F(x, y)=F(y,-x-y) \text {. }
$$

Thus if $F$ is of degree 3 and we put $F(x, y)=a x^{3}+b x^{2} y+c x y^{2}+d y^{2}$ then, by (74), $d=-a$ and $c=b-3 a$ and so the forms of degree 3 invariant under $C_{3}$ are $F(x, y)=a x^{3}+b x^{2} y+(b-3 a) x y^{2}-a y^{3}$ with $a$ and $b$ not both zero. If $F$ is invariant under $C_{4}$ then $F(x, y)=F(y,-x)$ and so $F$ must be of even degree and symmetric up to alternating signs. Thus, if $F$ is of degree 4 it has the form $F(x, y)=a x^{4}+b x^{3} y+c x^{2} y^{2}-b x y^{3}+a y^{4}$ with $a, b$, and $c$ not all zero. Next if $F$ is invariant under $C_{6}$ then $F(x, y)=F(-y, x+y)$ and $F$ is not of degree 3 or 5 while if it is of degree 4 it has the form $a\left(x^{2}+x y+y^{2}\right)^{2}$. The forms of degree 6 are

$$
\begin{aligned}
F(x, y)= & a x^{6}+b x^{5} y+c x^{4} y^{2}+(2 c+10 a-5 b) x^{3} y^{3} \\
& +(c+15 a-5 b) x^{2} y^{4}+(6 a-b) x y^{5}+a y^{6}
\end{aligned}
$$

with $a, b$, and $c$ not all zero.

$F$ is invariant under $D_{1}$ whenever the coefficients attached to odd powers of $y$ are zero, and is invariant under $D_{1}^{*}$ whenever $F$ is reciprocal. Further $F$ is invariant under $D_{2}$ whenever $F$ is of even degree and the coefficients attached to odd powers of $y$ are zero, while $F$ is invariant under $D_{2}^{*}$ whenever $F$ is of even degree and reciprocal. The forms invariant under $D_{3}$ are for degree 3, $a x y(x+y)$, degree 4, $a\left(x^{2}+x y+y^{2}\right)^{2}$, degree 5, axy $(x+y)\left(x^{2}+x y+y^{2}\right)$, all with $a \neq 0$, and for degree 6 ,

$$
F(x, y)=a x^{6}+3 a x^{5} y+c x^{4} y^{2}+(2 c-5 a) x^{3} y^{3}+c x^{2} y^{4}+3 a x y^{5}+a y^{6}
$$

with $a$ and $c$ not both zero. The forms invariant under $D_{3}^{*}$ are for degree 3, $\frac{a}{2}(x+2 y)(x-y)(2 x+y)$, degree $4, a\left(x^{2}+x y+y^{2}\right)^{2}$, degree 5, $\frac{a}{2}(x+2 y)(x-$ $y)(2 x+y)\left(x^{2}+x y+y^{2}\right)$, all with $a \neq 0$, and for degree 6 they are of the form (75) with $a$ and $c$ not both zero. The forms invariant under $D_{4}$ are reciprocal, of even degree, and the coefficients attached to odd powers of $y$ are zero. The forms of degree 4 are

$$
F(x, y)=a x^{4}+c x^{2} y^{2}+a y^{4},
$$

with $a$ and $c$ not both zero. Finally we consider forms $F$ invariant under $D_{6}$. Then $F(x, y)=F(y, x)=F(y,-x+y)$. There are no such forms of degree 3 or 5 and the only forms of degree 4 are $a\left(x^{2}-x y+y^{2}\right)^{2}$ with $a \neq 0$. The forms of degree 6 invariant under $D_{6}$ are

$$
F(x, y)=a x^{6}-3 a x^{5} y+c x^{4} y^{2}+(5 a-2 c) x^{3} y^{3}+c x^{2} y^{4}-3 a x y^{5}+a y^{6}
$$

with $a$ and $c$ not both zero. For such a form $F$, if $(x, y)$ is a solution of (1) then so also are $(y,-x+y),(-x+y,-x),(-x,-y),(-y, x-y),(x-y, x)$, $(y, x),(-x+y, y),(-x,-x+y),(-y,-x),(x-y,-y),(x, x-y)$. Further observe that these 12 solutions are distinct and primitive whenever $(x, y)$ 
is primitive and $(x, y)$ is different from $(1,0),(-1,0),(0,1),(0,-1)$, $(1,1),(-1,-1),(1,-1),(-1,1),(1,2),(-1,-2),(2,1)$, and $(-2,1)$.

Proof of Theorem 3. We shall first prove that $\nu^{*}(3) \geq 18$. Consider $F(x, y)=$ $x y(x+y)$. By the above discussion $F$ is invariant under $D_{3}$ and so whenever $(x, y)$ is a primitive solution of $(1),(y,-x-y),(-x-y, x),(y, x)$, $(-x-y, y)$, and $(x,-x-y)$ are also primitive solutions. If $x$ and $y$ are coprime integers and, as is readily checked, $(x, y)$ is not one of $(1,1)$, $(-1,-1),(1,-2),(-1,2),(2,-1)$, or $(-2,1)$ then the orbit of $(x, y)$ under $D_{3}$ consists of six distinct pairs.

For integers $a$ and $b$ we define $\varepsilon=\varepsilon(a, b)$ by

$$
\varepsilon= \begin{cases}1 & \text { if } 3 \mid 2 a+b \\ 0 & \text { otherwise }\end{cases}
$$

and we put

$$
f(a, b)=-a^{4}-2 a^{3} b+5 a^{2} b^{2}+6 a b^{3}+b^{4}
$$

Next we put

$$
\begin{gathered}
x(a, b)=\frac{a(a-b)}{2 \cdot 3^{\varepsilon}}, \quad y(a, b)=-\frac{(a+2 b)(a+b)}{2 \cdot 3^{\varepsilon}}, \\
u(a, b)=\frac{(2 a+b)(a+b)}{2 \cdot 3^{\varepsilon}}, \quad v(a, b)=\frac{b(a-b)}{2 \cdot 3^{\varepsilon}} \\
r(a, b)=\frac{b(2 a+b)+\sqrt{f(a, b)}}{2 \cdot 3^{\varepsilon}}, \quad s(a, b)=\frac{b(2 a+b)-\sqrt{f(a, b)}}{2 \cdot 3^{\varepsilon}} .
\end{gathered}
$$

We observe that

$$
\begin{aligned}
F(x(a, b), y(a, b)) & =F(u(a, b), v(a, b))=F(r(a, b), s(a, b)) \\
& =\frac{a b(a-b)(a+b)(a+2 b)(2 a+b)}{4 \cdot 3^{3 \varepsilon}} .
\end{aligned}
$$

We shall prove that if $a$ and $b$ are coprime odd integers for which $f(a, b)$ is the square of an integer then $(x(a, b), y(a, b)),(u(a, b), v(a, b))$, and $(r(a, b), s(a, b))$ are pairs of coprime integers. Further we shall show that there is a finite set of pairs such that if $(a, b)$ is not from that set then the orbits of $(x, y),(u, v)$, and $(r, s)$ under $D_{3}$ are disjoint. This will then establish that $\nu^{*}(3) \geq 18$ provided that we prove there are infinitely many pairs of coprime odd integers $(a, b)$ satisfying

$$
z^{2}=f(a, b)
$$

for some integer $z$, since, as is easily verified, for any pair of integers $(k, l)$ there are only finitely many pairs of coprime integers $(a, b)$ with $(x(a, b)$, $y(a, b)),(u(a, b), v(a, b))$, or $(r(a, b), s(a, b))$ equal to $(k, l)$.

Put $f(w)=f(1, w)$. Corresponding to the curve $Z^{2}=f(w)$ is the curve $t^{2}=4 s^{3}-g_{2} s-g_{3}$, where $g_{2}$ and $g_{3}$ are the invariants of the quartic $f$ (see, 
for instance, Chapter 16 of Mordell [27]). In particular, the map from the set of rational points $(s, t)$ on the curve

$$
t^{2}=4 s^{3}-\frac{49}{12} s+\frac{143}{216}
$$

minus the points $(17 / 12, \pm 5 / 2)$, to the set of rational points $(w, Z)$ on the curve $Z^{2}=f(w)$ given by

$$
w=\frac{t-5 / 2}{2 s-17 / 6}-\frac{3}{2} \text { and } Z=-\left(w+\frac{3}{2}\right)^{2}+2 s+\frac{17}{12}
$$

is injective. It follows that there are infinitely many rational solutions $(Z, w)$ of $Z^{2}=f(w)$ whenever there are infinitely many rational solutions $(s, t)$ of (78), or, equivalently, infinitely many rational solutions $(S, T)$ of

$$
T^{2}=S^{3}-1323 S+7722 .
$$

Observe that $P=(1057 / 16,29233 / 64)$ is a point on $(80)\left(P=4 P_{1}\right.$, where $P_{1}=(-21,162)$ ), so that by the theorem of Lutz and Nagell (see Corollary 7.2 of [38]), $P$ is a point of infinite order in the group of rational points of the elliptic curve given by $(80)$. This shows that there are infinitely many rational solutions $(Z, w)$ of $Z^{2}=f(w)$. For each solution we write $w=b / a$, where $a$ and $b$ are coprime integers and then clear denominators by multiplying through by $a^{4}$ to give a solution $\left(a^{2} Z, a, b\right)$ of (77) with $a$ and $b$ coprime integers. Thus there exist infinitely many pairs of coprime integers $(a, b)$ satisfying (77) and it remains to check that infinitely many of these pairs have $a$ and $b$ odd. First note that if $a$ and $b$ are coprime integers that give a solution of (77) and $a$ is odd and $b$ is even then

$$
z^{2} \equiv-a^{4} \equiv-1 \quad(\bmod 4)
$$

which is impossible. On the other hand, if $a$ and $b$ are coprime integers that give a solution of (77) and $a$ is even and $b$ is odd then, since

$$
f(a, b)=f(-a-b, b),
$$

$-a-b$ and $b$ are coprime odd integers that give a solution of (77). Thus there are infinitely many pairs $(a, b)$ of coprime odd integers that give a solution of (77).

We shall assume for the balance of the proof that $a$ and $b$ are coprime odd integers for which $f(a, b)$ is the square of an integer. We first check that then $x(a, b), y(a, b), u(a, b), v(a, b), r(a, b)$, and $s(a, b)$ are all integers. We remark that since $a$ and $b$ are odd, $a+b$ and $a-b$ are even. Further if 3 divides $2 a+b$ then 3 divides $a-b$ and $a+2 b$. Thus $x(a, b), y(a, b)$, $u(a, b)$, and $v(a, b)$ are integers. Since $a$ and $b$ are odd, $f(a, b)$ is odd and, since

$$
f(a, b)=(2 a+b)\left(4 a^{3}-3 a^{2} b+4 a b^{2}+b^{3}\right)-9 a^{4},
$$

$r(a, b)$ and $s(a, b)$ are integers. 
Now we shall show that $x(a, b)$ and $y(a, b)$ are coprime. We remark that since $a$ and $b$ are coprime, $a$ and $(a-b) /\left(2 \cdot 3^{\varepsilon}\right)$ are coprime and $(a+2 b) / 3^{\varepsilon}$ and $(a+b) / 2$ are coprime. Thus if $p$ is a prime that divides both $x$ and $y$ then either (i) $p \mid a$ and $p \mid(a+2 b) / 3^{\varepsilon}$, or (ii) $p \mid a$ and $p \mid(a+b) / 2$, or (iii) $p \mid(a-b) /\left(2 \cdot 3^{\varepsilon}\right)$ and $p \mid(a+2 b) / 3^{\varepsilon}$, or (iv) $p \mid(a-b) /\left(2 \cdot 3^{\varepsilon}\right)$ and $p \mid(a+b) / 2$. In case (i) $p \mid a$ and $p \mid 2 b$ so $p=2$, but $a$ is odd, which is a contradiction. In case (ii) $p \mid a$ and $p \mid b$, which is impossible. In case (iii) $p$ divides $3 a$ and $3 b$ so $p=3$. But one at least of $(a-b) /\left(2 \cdot 3^{\varepsilon}\right)$ and $(a+2 b) / 3^{\varepsilon}$ is not divisible by 3 and so case (iii) does not apply. Finally, in case (iv), $p \mid(a-b) / 2$ and $p \mid(a+b) / 2$, hence $p \mid(a, b)$, which is impossible. Thus $x(a, b)$ and $y(a, b)$ are coprime.

Next we show that $u(a, b)$ and $v(a, b)$ are coprime. Observe that $(2 a+$ $b) / 3^{\varepsilon}$ and $(a+b) / 2$ are coprime and that $b$ and $(a-b) /\left(2 \cdot 3^{\varepsilon}\right)$ are coprime. Thus if $p$ is a prime that divides both $u$ and $v$ then either $(\mathrm{i}) p \mid(2 a+b) / 3^{\varepsilon}$ and $p \mid b$, (ii) $p \mid(2 a+b) / 3^{\varepsilon}$ and $p \mid(a-b) /\left(2 \cdot 3^{\varepsilon}\right)$, (iii) $p \mid(a+b) / 2$ and $p \mid b$, or (iv) $p \mid(a+b) / 2$ and $p \mid(a-b) /\left(2 \cdot 3^{\varepsilon}\right)$. In case (i) $p \mid b$ and $p \mid 2 a$ so $p=2$, which contradicts the fact that $b$ is odd. In case (ii) $p \mid 3 a$ and $p \mid 3 b$, hence $p=3$. But one of $(2 a+b) / 3^{\varepsilon}$ and $(a-b) /\left(2 \cdot 3^{\varepsilon}\right)$ is not divisible by 3 and so (ii) does not hold. In case (iii) $p \mid a$ and $p \mid b$, which is impossible. Finally, in case (iv) $p \mid(a+b) / 2$ and $p \mid(a-b) / 2$, hence $p \mid a$ and $p \mid b$, which is a contradiction. Therefore $u(a, b)$ and $v(a, b)$ are coprime.

Finally we shall show that $r(a, b)$ and $s(a, b)$ are coprime. Suppose that $p$ is a prime that divides both $r$ and $s$. Then $p \mid r+s$ so $p \mid b(2 a+b) / 3^{\varepsilon}$. Since $b$ is odd, $b$ and $(2 a+b) / 3^{\varepsilon}$ are coprime. If $p \mid b$ then, since $p \mid r$, we see that $p \mid f(a, b)$ and hence that $p \mid a$, which is a contradiction. On the other harid, if $p \mid(2 a+b) / 3^{\varepsilon}$ then, since $p|r, p| f(a, b)$ and so by (81) $p \mid 9 a^{4}$. Thus $p=3$. If $3 \mid(2 a+b) / 3^{\varepsilon}$ then $3^{2} \mid 2 a+b$ and, since $a$ and $b$ are coprime, 3 does not divide $a$. From (81) we find that

$$
f(a, b)=(2 a+b)\left((2 a+b)\left(-7 a^{2}+2 a b+b^{2}\right)+18 a^{3}\right)-9 a^{4}
$$

and so 9 divides $f(a, b)$ but 27 does not divide $f(a, b)$. Since 9 divides $2 a+b$ we conclude that 3 exactly divides $b(2 a+b)+\sqrt{f(a, b)}$, hence 3 does not divide $r$. Therefore $r(a, b)$ and $s(a, b)$ are coprime.

To complete our proof that $\nu(F) \geq 18$, and hence that $\nu^{*}(3) \geq 18$, it suffices to show that apart from a finite set of pairs $(a, b)$ the orbits of $(x, y),(u, v)$, and $(r, s)$ under $D_{3}$ are disjoint. And a case by case analysis reveals that if $a$ and $b$ are odd and coprime and $(a, b)$ is different from $(1,-1),(-1,1)$, $(1,1),(-1,-1),(3,-1),(-3,1),(1,-5),(-1,5)$ then indeed the orbits are distinct as required.

Next we shall prove that $\nu^{*}(4) \geq 16$. We consider $F(x, y)=x^{4}+y^{4}$, which is invariant under $D_{4}$. Thus whenever $(x, y)$ is a solution of $(1)$, it follows that $(-x, y),(-x,-y),(x,-y),(y, x),(y,-x),(-y,-x)$, and $(-y, x)$ are also solutions. We now appeal to the parametric solution due to Euler of 
the equation $x^{4}+y^{4}=u^{4}+v^{4}$. He showed (see [18, p. 201]), that if

$$
\begin{aligned}
& x(t)=t^{7}+t^{5}-2 t^{3}+3 t^{2}+t, \\
& y(t)=t^{6}-3 t^{5}-2 t^{4}+t^{2}+1, \\
& u(t)=t^{7}+t^{5}-2 t^{3}-3 t^{2}+t, \\
& v(t)=t^{6}+3 t^{5}-2 t^{4}+t^{2}+1,
\end{aligned}
$$

then $x(t)^{4}+y(t)^{4}=u(t)^{4}+v(t)^{4}$. Put

$$
S(t)=-11 t^{5}+19 t^{4}+68 t^{3}+15 t^{2}-18 t-8
$$

and

$$
T(t)=11 t^{6}+14 t^{5}+7 t^{4}+15 t^{3}-24 t^{2}+8 t+66
$$

Then

$$
S(t) x(t)+T(t) y(t)=66 .
$$

If $t \equiv 0(\bmod 66)$ then $T(t) \equiv 0(\bmod 66), x(t) \equiv 0(\bmod 66)$, and $y(t) \equiv$ $1(\bmod 66)$, hence by $(82), x(t)$ and $y(t)$ are coprime. Next we put $M(t)=$ $-S(-t)$ and $N(t)=T(-t)$. Then, since $u(t)=-x(-t)$ and $v(t)=y(-t)$, we have

$$
M(t) u(t)+N(t) v(t)=66 .
$$

Again if $t \equiv 0(\bmod 66)$ then $N(t) \equiv 0(\bmod 66), u(t) \equiv 0(\bmod 66)$, and $v(t) \equiv 1(\bmod 66)$, hence by $(83), u(t)$ and $v(t)$ are coprime. Plainly the orbit of $(x(t), y(t))$ under $D_{4}$ does not contain $(u(t), v(t))$ for $t$ sufficiently large and thus $\nu^{*}(4) \geq 16$.

To prove that $\nu^{*}(5) \geq 6$ we merely note that $F(x, y)=x y(x+y)\left(x^{2}+x y+\right.$ $\left.y^{2}\right)$ is a form of degree 5 with nonzero discriminant that is invariant under $D_{3}$.

To prove that $\nu^{*}(6 k) \geq 12$ and $\nu^{*}(6 k+2) \geq 12$ for $k=1,2, \ldots$, it suffices to show that there exists a binary form with nonzero discriminant that is invariant under $D_{6}$ for these degrees. Let

$$
F_{c}(x, y)=x^{6}-3 x^{5} y+c x^{4} y^{2}+(5-2 c) x^{3} y^{3}+c x^{2} y^{4}-3 x y^{5}+y^{6}
$$

and put $f_{c}(x)=F_{c}(x, 1)$. Then the discriminant of $F_{c}$, and of $f_{c}$, is $-(4 c+3)^{3}(c-6)^{4}$. Thus the roots of $f_{c}$ are distinct provided that $c$ is an integer different from 6. Further if $c_{1}$ and $c_{2}$ are distinct integers then

$$
\begin{aligned}
F_{c_{1}}(x, y)-F_{c_{2}}(x, y) & =\left(c_{1}-c_{2}\right)\left(x^{4} y^{2}-2 x^{3} y^{3}+x^{2} y^{4}\right) \\
& =\left(c_{1}-c_{2}\right) x^{2} y^{2}(x-y)^{2} .
\end{aligned}
$$

Since $f_{c}(1)=1, f_{c_{1}}$ and $f_{c_{2}}$ have no roots in common. Further, by our earlier discussion $F_{c}(x, y)$ is invariant under $D_{6}$. Thus, for $k=1,2, \ldots$,

$$
\prod_{j=1}^{k} F_{6+j}(x, y)
$$


is a binary form of degree $6 k$ and nonzero discriminant that is invariant under $D_{6}$. Since $x^{2}-x y+y^{2}$ is invariant under $D_{6}$ and $f_{c}\left(e^{2 \pi i / 6}\right)=f_{c}\left(e^{-2 \pi i / 6}\right)=$ $c-6$,

$$
\left(x^{2}-x y+y^{2}\right) \prod_{j=1}^{k} F_{6+j}(x, y)
$$

is a binary form of nonzero discriminant of degree $6 k+2$ for $k=1,2, \ldots$ that is invariant under $D_{6}$. Thus $\nu^{*}(6 k) \geq 12$ and $\nu^{*}(6 k+2) \geq 12$ for $k=1,2, \ldots$.

Next we put $G_{c}(x, y)=F_{c}(-x, y)$ so that

$$
G_{c}(x, y)=x^{6}+3 x^{5} y+c x^{4} y^{2}+(2 c-5) x^{3} y^{3}+c x^{2} y^{4}+3 x y^{5}+y^{6} .
$$

Then, recall (75), $G_{c}$ is invariant under $D_{3}$. Put $g_{c}(x)=G_{c}(x, 1)$ so that $g_{c}(x)=f_{c}(-x)$. Then, as before, the roots of $g_{c}$ are distinct provided $c$ is an integer different from 6. Further if $c_{1}$ and $c_{2}$ are distinct integers then

$$
G_{c_{1}}(x, y)-G_{c_{2}}(x, y)=\left(c_{1}-c_{2}\right) x^{2} y^{2}(x+y)^{2} .
$$

Since $g_{c}(-1)=1, g_{c_{1}}$ and $g_{c_{2}}$ have no roots in common. Now both $x y(x+$ $y)$ and $x y(x+y)\left(x^{2}+x y+y^{2}\right)$ are invariant under $D_{3}$ and $g_{c}\left(e^{2 \pi i / 3}\right)=$ $g_{c}\left(e^{-2 \pi i / 3}\right)=c-6$. Thus, for $k=1,2, \ldots$,

$$
x y(x+y) \prod_{j=1}^{k} G_{6+j}(x, y)
$$

is a binary form of nonzero discriminant of degree $6 k+3$ that is invariant under $D_{3}$, and

$$
x y(x+y)\left(x^{2}+x y+y^{2}\right) \prod_{j=1}^{k} G_{6+j}(x, y)
$$

is a binary form of nonzero discriminant of degree $6 k+5$ that is invariant under $D_{3}$. Thus $\nu^{*}(6 k+3) \geq 6$ and $\nu^{*}(6 k+5) \geq 6$ for $k=1,2, \ldots$. Finally, observe that the binary form $x^{2 j}+y^{2 j}$ is invariant under $D_{4}$ for $j=1,2, \ldots$ and that the binary form $x^{j}+y^{j}$ is invariant under $D_{1}^{*}$ for $j=1,2, \ldots$ Thus $\nu^{*}(6 k+4) \geq 8$ and $\nu^{*}(6 k+1) \geq 2$ for $k=1,2, \ldots$.

\section{ON $S$-UNIT EQUATIONS}

Let $K$ be an algebraic number field of degree $d$, with discriminant $D_{K}$, and ring of integers $\mathscr{O}_{K}$. Let $M_{K}$ be the set of places (i.e., equivalence classes of multiplicative valuations) on $K$. A place $v$ is called finite if $v$ contains only non-Archimedean valuations and infinite otherwise. $K$ has only finitely many infinite places. Let $S$ be a finite subset of $M_{K}$, containing all infinite places. A number $\alpha \in K$ is called an $S$-unit if $|\alpha|_{v}=1$ for every valuation ||$_{v}$ from 
a place $v \in M_{K} \backslash S$. The $S$-units form a multiplicative group. Put $K \backslash\{0\}=K^{*}$ and let $\left(\alpha_{1}, \alpha_{2}, \alpha_{3}\right)$ be in $\left(K^{*}\right)^{3}$. The number of solutions of the equation

$$
\alpha_{1} u_{1}+\alpha_{2} u_{2}=\alpha_{3}
$$

in $S$-units $u_{1}$ and $u_{2}$ is finite (see Lang [20]). We say that two triples $\left(\alpha_{1}, \alpha_{2}, \alpha_{3}\right)$ and $\left(\beta_{1}, \beta_{2}, \beta_{3}\right)$ in $\left(K^{*}\right)^{3}$ are $S$-equivalent if there exist a permutation $\sigma$ of $(1,2,3)$, a $\mu \in K^{*}$, and $S$-units $\varepsilon_{1}, \varepsilon_{2}, \varepsilon_{3}$ such that

$$
\beta_{i}=\mu \varepsilon_{i} \alpha_{\sigma(i)} \text { for } i=1,2,3 .
$$

It is easy to check that if $\left(\alpha_{1}, \alpha_{2}, \alpha_{3}\right)$ and $\left(\beta_{1}, \beta_{2}, \beta_{3}\right)$ are $S$-equivalent then the equation $\beta_{1} u_{1}+\beta_{2} u_{2}=\beta_{3}$ in $S$-units $u_{1}$ and $u_{2}$ has the same number of solutions as (84). Next let $\mathfrak{p}_{1}, \ldots, \mathfrak{p}_{t}$ be the prime ideals corresponding to the finite places in $S$. For any $\alpha \in K^{*}$ the principal ideal $(\alpha)$ can be written uniquely as a product of two (not necessarily principal) ideals $\mathfrak{a}^{\prime}$ and $\mathfrak{a}^{\prime \prime}$, where $\mathfrak{a}^{\prime}$ is composed of $\mathfrak{p}_{1}, \ldots, \mathfrak{p}_{t}$ and $\mathfrak{a}^{\prime \prime}$ is composed solely of prime ideals different from $\mathfrak{p}_{1}, \ldots, \mathfrak{p}_{t}$. We put

$$
N_{S}(\alpha)=N_{K / \mathbb{Q}}\left(\mathfrak{a}^{\prime \prime}\right) \text {. }
$$

Recently, Evertse, Györy, Stewart, and Tijdeman [17] proved that almost all equivalence classes of $S$-unit equations of the form (84) have very few solutions and their result is our next lemma.

Lemma 7. Let $S$ be a finite subset of $M_{K}$ containing all infinite places. There exists a finite set $A$ of triples in $\left(\mathscr{O}_{K} \backslash\{0\}\right)^{3}$ with the following property: for each triple $\left(\alpha_{1}, \alpha_{2}, \alpha_{3}\right) \in\left(K^{*}\right)^{3}$ that is not $S$-equivalent to any of the triples from $A$, the number of solutions of (84) is at most two.

Proof. This is Theorem 1 of [17] together with the observation that we may take the triples in $A$ from $\left(\mathscr{C O}_{K} \backslash\{0\}\right)^{3}$.

$S$-unit equations are of great interest since the study of many Diophantine equations can be reduced to the study of certain associated $S$-unit equations. In the next section we shall make use of such a reduction to study the Thue-Mahler equation and the generalized Ramanujan-Nagell equation. We shall also appeal to an effective version of Lemma 7 established in [17].

Lemma 8. Let $S$ be a finite subset of $M_{K}$ of cardinality $s$, containing all infinite places. Suppose that the rational primes corresponding to the finite places in $S$ do not exceed $P(\geq 2)$. Let $B$ denote the set of triples $\left(\beta_{1}, \beta_{2}, \beta_{3}\right)$ in $\left(\mathscr{O}_{K} \backslash\{0\}\right)^{3}$ with

$$
\max \left(N_{S}\left(\beta_{1}\right), N_{S}\left(\beta_{2}\right), N_{S}\left(\beta_{3}\right)\right) \leq \exp \left(\left(C_{1} s\right)^{C_{2} s} P^{d+1}\right),
$$

where $C_{1}$ and $C_{2}$ are certain explicitly computed numbers depending only on $d$ and $\left|D_{K}\right|$. Then for each triple $\left(\alpha_{1}, \alpha_{2}, \alpha_{3}\right) \in\left(K^{*}\right)^{3}$ that is not $S$-equivalent to any of the triples in $B$, the number of solutions of (84) is at most $s+1$. 
Proof. If in inequality (85) we replace $\max \left(N_{S}\left(\beta_{1}\right), N_{S}\left(\beta_{2}\right), N_{S}\left(\beta_{3}\right)\right)$ by $\max \left(h\left(\beta_{1}\right), h\left(\beta_{2}\right), h\left(\beta_{3}\right)\right)$ we have Theorem 2 of [17]. The result now follows from the observation that for each $\beta$ in $\mathscr{O}_{K} \backslash\{0\}$,

$$
1 \leq N_{S}(\beta) \leq\left|N_{K / \mathbb{Q}}(\beta)\right| \leq h(\beta)^{d} .
$$

\section{Thue-Mahler and Ramanujan-Nagell equations}

Bombieri [3] has obtained an estimate for the number of primitive solutions of the Thue-Mahler equation (2) that is better with respect to the dependence on the degree $r$ than the estimate (3) of Evertse and yet is still independent of the coefficients of $F$. It follows from his result that, if $r$ is at least 6 and the discriminant of $F$ is nonzero then there are at most

$$
(4(t+1))^{2}(4 r)^{26(t+1)}
$$

primitive solutions of (2).

Let $h$ be a nonzero integer, let $t$ be a nonnegative integer, and let $p_{1}, \ldots, p_{t}$ be prime numbers. In this section we shall estimate the number of primitive solutions of the equation

$$
F(x, y)=h p_{1}^{k_{1}} \cdots p_{t}^{k_{t}}
$$

of course if $h=1$ we again obtain (2). We shall establish bounds for the number of solutions of (86) in coprime integers $x$ and $y$ and integers $k_{1}, \ldots, k_{t}$ under the assumption that $h$ is coprime with $p_{i}$ for $i=1, \ldots, t$ and sufficiently large. Our bounds are much sharper with respect to the parameter $t$ than the exponential dependence on $t$ of previous results.

Theorem 4. Let $F$ be a binary form with integer coefficients, content 1, degree $r(\geq 3)$, and nonzero discriminant $D$. Let $t$ be a nonnegative integer and let $p_{1}, \ldots, p_{t}$ be prime numbers of size at most $P(\geq 2)$. Let $h$ be a positive integer that is coprime with $p_{i}$ for $i=1, \ldots, t$. For $h$ sufficiently large the number of solutions of equation (86) in coprime integers $x$ and $y$ and integers $k_{1}, \ldots, k_{t}$ is at most

$$
4 r^{\omega(h)} \text {. }
$$

Further there exists a number $C$ that is effectively computable in terms of $r$ and $D$ such that if

$$
h>\exp \left((t+2)^{C(t+1)} P^{r^{3}}\right),
$$

then the number of solutions of (86) in coprime integers $x$ and $y$ and integers $k_{1}, \ldots, k_{t}$ is at most

$$
2(t+1) r^{3+\omega(h)}
$$

The most significant aspect of Theorem 4 is the dependence of the upper bounds (87) and (89) on the parameter $t$. Estimate (87), which is independent 
of $t$, applies for $h$ sufficiently large. However, since the proof of (87) depends upon Lemma 7 and hence upon the Thue-Siegel-Roth-Schmidt theorem it does not yield an effective estimate for how large $h$ must be. For this reason we have also given the slightly weaker estimate (89) that is linear in $t$ and holds subject to $h$ satisfying the effective estimate (88).

In fact, estimates as sharp as (87) and (89) do not apply for general $h$. Let $\varepsilon$ be a positive number and let $2=p_{1}, p_{2}, \ldots$ be the sequence of prime numbers. In [10] Erdös, Stewart, and Tijdeman proved that for every integer $r$ with $r \geq 2$ there exists a number $t_{0}(\varepsilon, r)$, which is effectively computable in terms of $\varepsilon$ and $r$ such that if $t$ is an integer with $t \geq t_{0}(\varepsilon, r)$ then there exists a monic polynomial $f$, with integer coefficients, degree $r$, and nonzero discriminant for which the equation

$$
f(x)=p_{1}^{k_{1}} \cdots p_{t}^{k_{t}}
$$

has at least

$$
\exp \left(\left(r^{2}-\varepsilon\right) t^{1 / r}(\log t)^{-(r-1) / r}\right)
$$

solutions in nonnegative integers $x, k_{1}, \ldots, k_{t}$. Recently Moree and Stewart [28] proved that, provided we replace $r^{2}-\varepsilon$ by $r-\varepsilon$ in (91), we may also suppose that $f$ is irreducible.

We remark that when $t=0$ estimate (87) gives a slight improvement, for $h$ sufficiently large, of the estimate (4) of Bombieri and Schmidt [5]. Further, if $r$ is odd then the proof of Theorem 4 allows one to replace $4 r^{\omega(h)}$ in (87) by $2 r^{\omega(h)}$ and similarly to eliminate the factor 2 in estimate (89).

Equation (90) is an example of a Ramanujan-Nagell equation. In [13] Evertse proved that if $f$ is a quadratic polynomial with integer coefficients and nonzero discriminant and $p_{1}, \ldots, p_{t}$ are distinct prime numbers then equation (90) has at most $3 \cdot 7^{6+4 t}$ solutions in integers $x, k_{1}, \ldots, k_{t}$. Let $h$ be a positive integer. Next we shall establish estimates for the number of solutions in integers $x, k_{1}, \ldots, k_{t}$ of the generalized Ramanujan-Nagell equation

$$
f(x)=h p_{1}^{k_{1}} \cdots p_{t}^{k_{t}} .
$$

Theorem 5. Let $f$ be a polynomial with integer coefficients, content 1 , leading coefficient $a$, degree $r(\geq 2)$, and nonzero discriminant $D$. Let $t$ be a nonnegative integer and let $p_{1}, \ldots, p_{t}$ be prime numbers of size at most $P(\geq 2)$. Let $h$ be a positive integer that is coprime with $p_{i}$ for $i=1, \ldots, t$. For $h$ sufficiently large the number of solutions of (92) in integers $x$ and $k_{1}, \ldots, k_{t}$ is at most

$$
2 r^{\omega(h)}
$$

Further there exists a number $C$, which is effectively computable in terms of a, $r$, and $D$, such that if

$$
h>\exp \left((t+2)^{C(t+1)} P^{r^{2}}\right)
$$


then the number of solutions of (92) in integers $x$ and $k_{1}, \ldots, k_{t}$ is at most $(t+1) r^{2+\omega(h)}$.

Finally we mention that Evertse and Györy [14] have also applied the estimates for the number of solutions of $S$-unit equations from [17] to bound the number of solutions of equations such as (86). Let $S=\left\{p_{1}, \ldots, p_{t}\right\}$ be a set of primes. Two binary forms $F$ and $G$ are $S$-equivalent if $G(x, y)=$ $e f^{-1} F(a x+b y, c x+d y)$ for certain integers $a, b, c, d, e, f$ with $|a d-b c|$, $|e|$, and $|f|$ composed of primes from $S$. For any algebraic number field $L$ and integer $r \geq 3$ let $A(r, L)$ be the set of binary forms of degree $r$ with integer coefficients that factorize into linear forms in $L[x, y]$ and whose factorization contains at least three pairwise linearly independent linear forms. Evertse and Györy show, for instance, that the set of forms in $A(r, L)$ for which (86) has more than $2(r, 2)$ solutions is contained in the union of a finite collection of $S$-equivalence classes.

\section{Proof of THEOREM 4}

Since $D \neq 0, F(x, y)$ has at most a single power of $x$ and at most a single power of $y$ in any factorization in $\mathbb{C}[x, y]$. Thus we may factor $F$ as

$$
F(x, y)=a x^{\delta_{1}}\left(x-\alpha_{1+\delta_{1}} y\right) \cdots\left(x-\alpha_{r-\delta_{2}} y\right) y^{\delta_{2}}
$$

and

$$
F(x, y)=b y^{\delta_{2}}\left(y-\gamma_{1+\delta_{2}} x\right) \cdots\left(y-\gamma_{r-\delta_{1}} x\right) x^{\delta_{1}},
$$

where $a$ and $b$ are nonzero integers, $\delta_{1}$ and $\delta_{2}$ are from $\{0,1\}$, and $\gamma_{r+1-j}=$ $\alpha_{j}^{-1}$ for $j=1+\delta_{1}, \ldots, r-\delta_{2}$. Put $K=\mathbb{Q}\left(\alpha_{1+\delta_{1}}, \ldots, \alpha_{r-\delta_{2}}\right)$ and let $\mathscr{O}_{K}$ denote the ring of algebraic integers of $K$. Let $q$ be a prime number and let $q$ be a prime ideal in $\mathscr{C}_{K}$ lying above $q$. For each $\alpha \in K^{*}$ we define ord $\alpha$ to be the exponent of $\mathfrak{q}$ in the prime ideal decomposition of the fractional ideal of $K$ generated by $\alpha$. We shall suppose that

$$
\operatorname{ord}_{\mathfrak{q}} \alpha_{1+\delta_{1}} \geq \cdots \geq \operatorname{ord}_{\mathfrak{q}} \alpha_{w} \geq 0>\operatorname{ord}_{\mathfrak{q}} \alpha_{w+1} \geq \cdots \geq \operatorname{ord}_{\mathfrak{q}} \alpha_{r-\delta_{2}},
$$

where $\delta_{1} \leq w \leq r-\delta_{2}$. Since $F$ has content 1 ,

$$
\operatorname{ord}_{\mathfrak{q}} a+\operatorname{ord}_{\mathfrak{q}} \alpha_{w+1}+\cdots+\operatorname{ord}_{\mathfrak{q}} \alpha_{r-\delta_{2}}=0 .
$$

Put $u=r-\delta_{2}$ and if $\delta_{1}=1$ put $\alpha_{1}=0$. Then, from (94) we have

$$
F(x, y)=a\left(x-\alpha_{1} y\right) \cdots\left(x-\alpha_{u} y\right) y^{\delta_{2}} .
$$

Similarly put $v=r-\delta_{1}$ and if $\delta_{2}=1$ put $\gamma_{1}=0$ so that, by (95),

$$
F(x, y)=b\left(y-\gamma_{1} x\right) \cdots\left(y-\gamma_{v} x\right) x^{\delta_{1}} .
$$

We shall now consider the tuples of the form

$$
\left(\operatorname{ord}_{\mathfrak{q}} x^{\delta_{1}}, \operatorname{ord}_{\mathfrak{q}}\left(x-\alpha_{1+\delta_{1}} y\right), \ldots, \operatorname{ord}_{\mathfrak{q}}\left(x-\alpha_{r-\delta_{2}} y\right), \operatorname{ord}_{\mathfrak{q}} y^{\delta_{2}}\right),
$$


where $(x, y)$ yields a primitive solution of (86). If $q$ does not divide $h p_{1} \cdots p_{t}$ then (98) is determined independently of $x$ and $y$. For if $q \nmid a$ then by (86) it is $(0,0, \ldots, 0)$ whereas if $q \mid a$ then by (86), (96), and (97) it is

$$
\left(0, \ldots, 0, \operatorname{ord}_{\mathfrak{q}} \alpha_{w+1}, \ldots, \operatorname{ord}_{\mathfrak{q}} \alpha_{r-\delta_{2}}, 0\right) .
$$

We shall now show that if $q \mid h$ then there are at most $r$ positive tuples of the form (98) whenever $x$ and $y$ give coprime solutions of (86).

We first suppose that $(x, y)$ yields a solution of $(86)$ in coprime integers and that $q \mid h$ and $q \nmid y$. We now choose an integer $l$, with $1 \leq l \leq u$, for which

$$
\operatorname{ord}_{\mathfrak{q}}\left(x-\alpha_{l} y\right)=\max _{1 \leq i \leq u} \operatorname{ord}_{\mathfrak{q}}\left(x-\alpha_{i} y\right) \text {. }
$$

Since $q \nmid y, \operatorname{ord}_{\mathfrak{q}} \alpha_{j}=\operatorname{ord}_{q} \alpha_{j} y<0 \leq \operatorname{ord}_{q} x$ and hence $\operatorname{ord}_{q}\left(x-\alpha_{j} y\right)=$ $\operatorname{ord}_{q} \alpha_{j}$ for $j=w+1, \ldots, u$. Since $q \mid h$ we conclude from (86) that $1 \leq l \leq$ $w$. Observe that, for $j=1, \ldots, u$,

$$
x-\alpha_{j} y=x-\alpha_{l} y+\left(\alpha_{l}-\alpha_{j}\right) y
$$

hence, since $q \nmid y$,

$$
\operatorname{ord}_{\mathfrak{q}}\left(x-\alpha_{j} y\right)=\min \left(\operatorname{ord}_{\mathfrak{q}}\left(x-\alpha_{l} y\right), \operatorname{ord}_{\mathfrak{q}}\left(\alpha_{l}-\alpha_{j}\right)\right) .
$$

Further, by (86) we have

$$
\operatorname{ord}_{\mathfrak{q}} a+\operatorname{ord}_{\mathfrak{q}}\left(x-\alpha_{1} y\right)+\cdots+\operatorname{ord}_{\mathfrak{q}}\left(x-\alpha_{u} y\right)=\operatorname{ord}_{\mathfrak{q}} h .
$$

Equations (99) and (100) determine $\operatorname{ord}_{\mathfrak{q}}\left(x-\alpha_{l} y\right)$ and hence also $\operatorname{ord}_{\mathfrak{q}}\left(x-\alpha_{j} y\right)$ for $j=1, \ldots, u$. Thus there are at most $w$ possible tuples (98) that can arise from primitive solutions $(x, y)$ of (86) for which $q \nmid y$ and $q \mid h$.

Suppose now that $(x, y)$ is a primitive solution of (86) for which $q \mid y$ and $q \mid h$. Then, since $x$ and $y$ are coprime, $q \nmid x$. Since $\gamma_{r+1-j}=\alpha_{j}^{-1}$ for $j=1+\delta_{1}, \ldots, r-\delta_{2}$ we have, by (96),

$$
\operatorname{ord}_{q} \gamma_{1+\delta_{2}} \geq \cdots \geq \operatorname{ord}_{q} \gamma_{r-w}>0 \geq \operatorname{ord}_{q} \gamma_{r-w+1} \geq \cdots \geq \operatorname{ord}_{q} \gamma_{r-\delta_{1}} .
$$

Further, since $F$ has content 1 ,

$$
\operatorname{ord}_{\mathfrak{q}} b+\operatorname{ord}_{\mathfrak{q}} \gamma_{r-w+1}+\cdots+\operatorname{ord}_{\mathfrak{q}} \gamma_{r-\delta_{1}}=0 .
$$

Now $q \nmid x$ and $q \mid y$ so $\operatorname{ord}_{\mathfrak{q}} \gamma_{j}=\operatorname{ord}_{\mathfrak{q}} \gamma_{j} x \leq 0<\operatorname{ord}_{\mathfrak{q}} y$ and hence $\operatorname{ord}_{\mathfrak{q}}\left(y-\gamma_{j} x\right)=\operatorname{ord}_{\mathfrak{q}} \gamma_{j}$ for $j=r-w+1, \ldots, r-\delta_{1}$. We now choose $k$ so that

$$
\operatorname{ord}_{\mathfrak{q}}\left(y-\gamma_{k} x\right)=\max _{1 \leq i \leq v} \operatorname{ord}_{\mathfrak{q}}\left(y-\gamma_{i} x\right)
$$

Since $q \mid h$ we see from (86) and (101) that $1 \leq k \leq r-w$. Further, for $j=1, \ldots, v$,

$$
\operatorname{ord}_{\mathfrak{q}}\left(y-\gamma_{j} x\right)=\min \left(\operatorname{ord}_{\mathfrak{q}}\left(y-\gamma_{k} x\right), \operatorname{ord}_{\mathfrak{q}}\left(\gamma_{k}-\gamma_{j}\right)\right) .
$$


We also have, from (86),

$$
\operatorname{ord}_{\mathfrak{q}} b+\operatorname{ord}_{\mathfrak{q}}\left(y-\gamma_{1} x\right)+\cdots+\operatorname{ord}_{\mathfrak{q}}\left(y-\gamma_{v} x\right)=\operatorname{ord}_{\mathfrak{q}} h .
$$

As before, (102) and (103) determine $\operatorname{ord}_{\mathfrak{q}}\left(y-\gamma_{k} x\right)$, hence $\operatorname{ord}_{\mathfrak{q}}\left(y-\gamma_{j} x\right)$ for $j=1, \ldots, v$, and thus in turn they determine (98).

Therefore if $(x, y)$ gives a primitive solution of (86) and $q \mid h$ then (98) is one of at most $(r-w)+w=r$ possible tuples, whereas if $q+h p_{1} \cdots p_{t}$ the tuple $(98)$ is uniquely determined. Since $K$ is Galois over $\mathbb{Q}$, all prime ideals of $\mathscr{O}_{K}$ lying over $q$ are conjugate. Every automorphism of $K$ induces a permutation of $\left(\alpha_{1+\delta_{1}}, \ldots, \alpha_{r-\delta_{2}}\right)$ and thus corresponding to each tuple (98) there is, for each prime ideal $q^{\prime}$ lying over $q$ in $\mathscr{O}_{K}$, a unique tuple of the form (98) but with $\mathfrak{q}$ replaced by $\mathfrak{q}^{\prime}$.

For notational ease we write

$$
F(x, y)=a \prod_{i=1}^{r}\left(\theta_{i} x-\alpha_{i} y\right),
$$

where $\theta_{i}=1$ for $i=1, \ldots, r$ except when $\delta_{2}=1$ in which case $\theta_{r}=0$ and $\alpha_{r}=-1$. Let $S$ denote the set of infinite places in $K$ together with those finite places that correspond to a prime ideal in $\mathscr{O}_{K}$ that divides an ideal generated by $p_{j}$ for $j=1, \ldots, t$. Let $\left\{\left(x_{1}, y_{1}\right), \ldots,\left(x_{n}, y_{n}\right)\right\}$ be a set of pairs of coprime integers that give solutions of (97) and suppose that the set is maximal subject to the constraint that whenever $j \neq i$ the tuple

$$
\left(\frac{\theta_{1} x_{j}-\alpha_{1} y_{j}}{\theta_{1} x_{i}-\alpha_{1} y_{i}}, \ldots, \frac{\theta_{r} x_{j}-\alpha_{r} y_{j}}{\theta_{r} x_{i}-\alpha_{r} y_{i}}\right)
$$

is not a tuple of $S$-units. Then, by the preceding discussion, $n \leq r^{\omega(h)}$.

Let $x$ and $y$ be coprime integers that give a solution of (86). Then there is an integer $j$ with $1 \leq j \leq n$ such that the tuple (104) is a tuple of $S$-units when we replace $x_{i}, y_{i}$ by $x, y$ respectively. We may assume, without loss of generality, that

$$
\begin{aligned}
& N_{S}\left(\left(\theta_{1} x_{j}-\alpha_{1} y_{j}\right)\left(\theta_{2} x_{j}-\alpha_{2} y_{j}\right)\left(\theta_{3} x_{j}-\alpha_{3} y_{j}\right)\right) \\
& \quad \geq N_{S}\left(\left(\theta_{i_{1}} x_{j}-\alpha_{i_{1}} y_{j}\right)\left(\theta_{i_{2}} x_{j}-\alpha_{i_{2}} y_{j}\right)\left(\theta_{i_{3}} x_{j}-\alpha_{i_{3}} y_{j}\right)\right)
\end{aligned}
$$

for all triples $\left(i_{1}, i_{2}, i_{3}\right)$ with $1 \leq i_{1}<i_{2}<i_{3} \leq r$. Thus, by (86),

$$
N_{S}\left(\left(\theta_{1} x_{j}-\alpha_{1} y_{j}\right)\left(\theta_{2} x_{j}-\alpha_{2} y_{j}\right)\left(\theta_{3} x_{j}-\alpha_{3} y_{j}\right)\right) \geq\left(N_{S}(h / a)\right)^{3 / r} \text {. }
$$

Let $K_{1}=\mathbb{Q}\left(\alpha_{1}, \alpha_{2}, \alpha_{3}\right)$ and let $S_{1}$ denote the set of infinite places in $K_{1}$ together with those finite places that correspond to a prime ideal in $\mathscr{O}_{K_{1}}$ that divides an ideal generated by $p_{j}$ for $j$ with $1 \leq j \leq t$. Let $d_{1}$ denote the degree of $K_{1}$ over $\mathbb{Q}$. It follows from (105) that

$$
N_{S_{1}}\left(\left(\theta_{1} x_{j}-\alpha_{1} y_{j}\right)\left(\theta_{2} x_{j}-\alpha_{2} y_{j}\right)\left(\theta_{3} x_{j}-\alpha_{3} y_{j}\right)\right) \geq\left(N_{S_{1}}(h / a)\right)^{3 / r} .
$$


Put

$$
\begin{aligned}
& \lambda=a\left(\theta_{2} \alpha_{3}-\theta_{3} \alpha_{2}\right)\left(\theta_{1} x_{j}-\alpha_{1} y_{j}\right), \\
& \eta=a\left(\theta_{3} \alpha_{1}-\theta_{1} \alpha_{3}\right)\left(\theta_{2} x_{j}-\alpha_{2} y_{j}\right),
\end{aligned}
$$

and

$$
\tau=a\left(\theta_{2} \alpha_{1}-\theta_{1} \alpha_{2}\right)\left(\theta_{3} x_{j}-\alpha_{3} y_{j}\right) .
$$

Plainly $\lambda, \eta$, and $\tau$ are in $\mathscr{O}_{K_{1}} \backslash\{0\}$. Further

$$
\begin{aligned}
& a\left(\theta_{2} \alpha_{3}-\theta_{3} \alpha_{2}\right)\left(\theta_{1} x-\alpha_{1} y\right)=\lambda u_{1}, \\
& a\left(\theta_{3} \alpha_{1}-\theta_{1} \alpha_{3}\right)\left(\theta_{2} x-\alpha_{2} y\right)=\eta u_{2},
\end{aligned}
$$

and

$$
a\left(\theta_{2} \alpha_{1}-\theta_{1} \alpha_{2}\right)\left(\theta_{3} x-\alpha_{3} y\right)=\tau u_{3},
$$

where $u_{1}, u_{2}$, and $u_{3}$ are $S_{1}$-units. But then

$$
\begin{aligned}
& \frac{\left(\theta_{2} \alpha_{3}-\theta_{3} \alpha_{2}\right)\left(\theta_{1} x-\alpha_{1} y\right)}{\left(\theta_{2} \alpha_{1}-\theta_{1} \alpha_{2}\right)\left(\theta_{3} x-\alpha_{3} y\right)}=\frac{\lambda}{\tau} U_{1}, \\
& \frac{\left(\theta_{3} \alpha_{1}-\theta_{1} \alpha_{3}\right)\left(\theta_{2} x-\alpha_{2} y\right)}{\left(\theta_{2} \alpha_{1}-\theta_{1} \alpha_{2}\right)\left(\theta_{3} x-\alpha_{3} y\right)}=\frac{\eta}{\tau} U_{2},
\end{aligned}
$$

where $U_{1}$ and $U_{2}$ are $S_{1}$-units, and so

$$
\lambda U_{1}+\eta U_{2}=\tau .
$$

Further, by (107), each pair of $S_{1}$-integers $\left(U_{1}, U_{2}\right)$ determines at most two pairs of coprime integers $(x, y),((x, y),(-x,-y))$.

Suppose that $\left(\beta_{1}, \beta_{2}, \beta_{3}\right)$ is a triple in $\left(\mathscr{O}_{K_{1}} \backslash\{0\}\right)^{3}$ that is $S_{1}$-equivalent to $(\lambda, \eta, \tau)$. Then there exist a permutation $\sigma$ of $(1,2,3)$, a $\mu$ in $K_{1}^{*}$, and $S_{1}$-units $\varepsilon_{1}, \varepsilon_{2}, \varepsilon_{3}$ such that $\mu \varepsilon_{1} \lambda=\beta_{\sigma(1)}, \mu \varepsilon_{2} \eta=\beta_{\sigma(2)}$, and $\mu \varepsilon_{3} \tau=\beta_{\sigma(3)}$. We shall now show that the maximum of $N_{S_{1}}\left(\beta_{1}\right), N_{S_{1}}\left(\beta_{2}\right)$, and $N_{S_{1}}\left(\beta_{3}\right)$ is large. To this end, let $\mathfrak{p}$ be a prime ideal of $\mathscr{O}_{K_{1}} \backslash\{0\}$ and put $a_{1}=\operatorname{ord}_{\mathfrak{p}} a$ and $b_{1}=\operatorname{ord}_{\mathfrak{p}}\left(\theta_{2} \alpha_{1}-\theta_{1} \alpha_{2}\right)$. Let $\left(i_{1}, \ldots, i_{r}\right)$ be a permutation of $(1, \ldots, r)$ for which

$$
\operatorname{ord}_{\mathfrak{p}} \alpha_{i_{1}} \leq \operatorname{ord}_{\mathfrak{p}} \alpha_{i_{2}} \leq \cdots \leq \operatorname{ord}_{\mathfrak{p}} \alpha_{i_{r}}
$$

and let $g$ be that integer with $0 \leq g \leq r$ for which

$$
\operatorname{ord}_{\mathfrak{p}} \alpha_{i_{1}} \leq \cdots \leq \operatorname{ord}_{\mathfrak{p}} \alpha_{i_{g}}<0 \leq \operatorname{ord}_{\mathfrak{p}} \alpha_{i_{g+1}} \leq \cdots \leq \operatorname{ord}_{\mathfrak{p}} \alpha_{i_{r}}
$$

Since $F$ has content 1 we have

$$
-a_{1}=\operatorname{ord}_{\mathfrak{p}} \alpha_{i_{1}}+\cdots+\operatorname{ord}_{\mathfrak{p}} \alpha_{i_{g}} .
$$

Thus

$$
\begin{aligned}
& \quad \sum_{i<j, \operatorname{ord}_{\mathfrak{p}}\left(\theta_{j} \alpha_{i}-\theta_{i} \alpha_{j}\right)<0} \operatorname{ord}_{\mathfrak{p}}\left(\theta_{j} \alpha_{i}-\theta_{i} \alpha_{j}\right) \\
& \geq(n-1) \operatorname{ord}_{\mathfrak{p}} \alpha_{i_{1}}+(n-2) \operatorname{ord}_{\mathfrak{p}} \alpha_{i_{2}}+\cdots+(n-g) \operatorname{ord}_{\mathfrak{p}} \alpha_{i_{g}} \\
& \geq-(n-1) a_{1} .
\end{aligned}
$$


Therefore, since

$$
D=a^{2 n-2} \prod_{i<j}\left(\theta_{j} \alpha_{i}-\theta_{i} \alpha_{j}\right)^{2},
$$

we find that

$$
\begin{aligned}
& \operatorname{ord}_{\mathfrak{p}}\left(\theta_{1} \alpha_{2}-\theta_{2} \alpha_{1}\right)+\max \left(\operatorname{ord}_{\mathfrak{p}}\left(\theta_{2} \alpha_{3}-\theta_{3} \alpha_{2}\right), \operatorname{ord}_{\mathfrak{p}}\left(\theta_{3} \alpha_{1}-\theta_{1} \alpha_{3}\right)\right) \\
& \quad \leq \frac{1}{2} \operatorname{ord}_{\mathfrak{p}} D
\end{aligned}
$$

We have

$$
\theta_{2}\left(\theta_{1} x_{j}-\alpha_{1} y_{j}\right)-\theta_{1}\left(\theta_{2} x_{j}-\alpha_{2} y_{j}\right)=\left(\theta_{1} \alpha_{2}-\theta_{2} \alpha_{1}\right) y_{j}
$$

and

$$
\alpha_{2}\left(\theta_{1} x_{j}-\alpha_{1} y_{j}\right)-\alpha_{1}\left(\theta_{2} x_{j}-\alpha_{2} y_{j}\right)=\left(\theta_{1} \alpha_{2}-\theta_{2} \alpha_{1}\right) x_{j} .
$$

Thus, by (109) and (111),

$$
\min \left(\operatorname{ord}_{\mathfrak{p}}\left(\theta_{1} x_{j}-\alpha_{1} y_{j}\right), \operatorname{ord}_{\mathfrak{p}}\left(\theta_{2} x_{j}-\alpha_{2} y_{j}\right)\right) \leq a_{1}+b_{1}+\operatorname{ord}_{\mathfrak{p}} y_{j},
$$

while, by (109) and (112),

$$
\min \left(\operatorname{ord}_{\mathfrak{p}}\left(\theta_{1} x_{j}-\alpha_{1} y_{j}\right), \operatorname{ord}_{\mathfrak{p}}\left(\theta_{2} x_{j}-\alpha_{2} y_{j}\right)\right) \leq a_{1}+b_{1}+\operatorname{ord}_{\mathfrak{p}} x_{j}
$$

Since $x_{j}$ and $y_{j}$ are coprime we conclude that

$$
\min \left(\operatorname{ord}_{\mathfrak{p}}\left(\theta_{1} x_{j}-\alpha_{1} y_{j}\right), \operatorname{ord}_{\mathfrak{p}}\left(\theta_{2} x_{j}-\alpha_{2} y_{j}\right)\right) \leq a_{1}+b_{1} .
$$

Therefore

$$
\begin{aligned}
& \min \left(\operatorname{ord}_{\mathfrak{p}} \lambda, \operatorname{ord}_{\mathfrak{p}} \eta\right) \\
& \quad \leq 2 a_{1}+b_{1}+\max \left(\operatorname{ord}_{\mathfrak{p}}\left(\theta_{2} \alpha_{3}-\theta_{3} \alpha_{2}\right), \operatorname{ord}_{\mathfrak{p}}\left(\theta_{3} \alpha_{1}-\theta_{1} \alpha_{3}\right)\right)
\end{aligned}
$$

Thus, by (110) and (115),

$$
\min \left(\operatorname{ord}_{\mathfrak{p}} \lambda, \operatorname{ord}_{\mathfrak{p}} \eta, \operatorname{ord}_{\mathfrak{p}} \tau\right) \leq 2 a_{1}+\frac{1}{2} \operatorname{ord}_{\mathfrak{p}} D
$$

Accordingly,

$$
N_{S_{1}}\left(\beta_{1} \beta_{2} \beta_{3}\right) \geq N_{S_{1}}(\lambda \eta \tau) \cdot\left(N_{S_{1}}\left(a^{2} D\right)\right)^{-3} .
$$

Since $a^{3}\left(\theta_{2} \alpha_{3}-\theta_{3} \alpha_{2}\right)\left(\theta_{3} \alpha_{1}-\theta_{1} \alpha_{3}\right)\left(\theta_{1} \alpha_{2}-\theta_{2} \alpha_{1}\right)$ is in $\mathscr{O}_{K_{1}} \backslash\{0\}$, it follows from (106) and (116) that

$$
N_{S_{1}}\left(\beta_{1} \beta_{2} \beta_{3}\right) \geq\left(N_{S_{1}}(h / a)\right)^{3 / r}\left(N_{S_{1}}\left(a^{2} D\right)\right)^{-3} \geq h^{3 d_{1} / r}\left(N_{S_{1}}\left(a^{3} D\right)\right)^{-3} .
$$

Thus

$$
\max \left(N_{S_{1}}\left(\beta_{1}\right), N_{S_{1}}\left(\beta_{2}\right), N_{S_{1}}\left(\beta_{3}\right)\right) \geq h^{d_{1} / r}\left|a^{3} D\right|^{-d_{1}} .
$$

On the other hand, by Lemma 7 , there is a finite set $A$ of triples in $\left(\mathscr{O}_{K_{1}} \backslash\{0\}\right)^{3}$ such that if $\left(\delta_{1}, \delta_{2}, \delta_{3}\right)$ is in $\left(K_{1}^{*}\right)^{3}$ and the $S_{1}$-unit equation $\delta_{1} u_{1}+\delta_{2} u_{2}=\delta_{3}$ has more than two solutions in $S_{1}$-units $u_{1}$ and $u_{2}$ then $\left(\delta_{1}, \delta_{2}, \delta_{3}\right)$ is $S_{1}$ equivalent to one of the triples from $A$. Thus, by (117), if $h$ is sufficiently 
large then the equation (108) determined by the triple $(\lambda, \eta, \tau)$ has at most two solutions in $S_{1}$-units $U_{1}$ and $U_{2}$ and these solutions arise from at most four primitive solutions of (86), in fact at most two primitive solutions if $r$ is odd. Since there are at most $n$ such triples $(\lambda, \eta, \tau)$ the number of solutions of (86) in coprime integers $x, y$ and integers $k_{1}, \ldots, k_{t}$ is at most $4 n$, hence at most $4 r^{w(h)}$ for $h$ sufficiently large.

To prove (89) we apply Lemma 8 with $K=K_{1}, S=S_{1}$, and $d=d_{1}$. Note that

$$
\left|S_{1}\right|+1 \leq d_{1} t+d_{1}+1 \leq r(r-1)(r-2)(t+1)+1<r^{3}(t+1) .
$$

Further, there exist numbers $C_{1}$ and $C_{2}$ that are explicitly computable in terms of $d_{1}$ and $\left|D_{K_{1}}\right|$ such that if $\left(\alpha_{1}, \alpha_{2}, \alpha_{3}\right)$ is a triple in $\left(K_{1}^{*}\right)^{3}$ that is not $S_{1}$ equivalent to a triple $\left(\nu_{1}, \nu_{2}, \nu_{3}\right)$ in $\left(\mathscr{O}_{K_{1}} \backslash\{0\}\right)^{3}$ with

$$
\max \left(N_{S_{1}}\left(\nu_{1}\right), N_{S_{1}}\left(\nu_{2}\right), N_{S_{1}}\left(\nu_{3}\right)\right) \leq \exp \left(C_{1}(t+1)^{C_{2}(t+1)} P^{d_{1}+1}\right),
$$

then equation (84) has at most $\left|S_{1}\right|+1$ solutions in $S_{1}$-units $u_{1}$ and $u_{2}$. Thus, by (117), provided

$$
h>\left(|a|^{3}|D|\right)^{r} \exp \left(r C_{1}(t+1)^{C_{2}(t+1)} P^{d_{1}+1}\right),
$$

equation (108) has at most $\left|S_{1}\right|+1$, hence at most $r^{3}(t+1)$, solutions in $S_{1}$ units $U_{1}$ and $U_{2}$ and these solutions arise from at most $2 r^{3}(t+1)$ primitive solutions of (86). Since the number or primitive solutions of $(86)$ is unchanged when we replace $F$ by $F_{A}$ for any $A$ in $\operatorname{GL}(2, \mathbb{Z})$, we may suppose that $|a|$ is minimal. It follows from Theorem 1 of Evertse and Györy [15] that we may suppose that $|a|$ is less than a number that is effectively computable in terms of $r$ and $D$ only. Therefore there is a number $C_{3}$, which is effectively computable in terms of $r$ and $D$, such that if

$$
h>\exp \left((t+2)^{C_{3}(t+1)} P^{r^{3}}\right),
$$

then (86) has at most $2(t+1) r^{3+w(h)}$ solutions in coprime integers $x, y$ and integers $k_{1}, \ldots, k_{t}$.

\section{Proof of Theorem 5}

Let $f(x)=a\left(x-\alpha_{1}\right) \cdots\left(x-\alpha_{r}\right)$, let $K=\mathbb{Q}\left(\alpha_{1}, \ldots, \alpha_{r}\right)$, and let $S$ denote the set of infinite places in $K$ together with those finite places that correspond to a prime ideal in $\mathscr{O}_{K}$ that divides an ideal generated by $p_{j}$ for $j=1, \ldots, t$. Let $\left\{x_{1}, \ldots, x_{n}\right\}$ be integers that give solutions of (92) and suppose that the set is maximal subject to the constraint that whenever $j \neq i$ the tuple

$$
\left(\frac{x_{j}-\alpha_{1}}{x_{i}-\alpha_{1}}, \ldots, \frac{x_{j}-\alpha_{r}}{x_{i}-\alpha_{r}}\right)
$$

is not a tuple of $S$-units. Then, as in the proof of Theorem $4, n \leq r^{w(h)}$. 
Let $x$ be an integer that gives a solution of (92). Then there is an integer $j$ with $1 \leq j \leq n$ such that the tuple (118) is a tuple of $S$-units when we replace $x_{i}$ by $x$. We may assume, without loss of generality, that

$$
N_{S}\left(\left(x_{j}-\alpha_{1}\right)\left(x_{j}-\alpha_{2}\right)\right) \geq N_{S}\left(\left(x_{j}-\alpha_{i_{1}}\right)\left(x_{j}-\alpha_{i_{2}}\right)\right)
$$

for all pairs $\left(i_{1}, i_{2}\right)$ with $1 \leq i_{1}<i_{2} \leq r$. Thus, by (92),

$$
N_{S}\left(\left(x_{j}-\alpha_{1}\right)\left(x_{j}-\alpha_{2}\right)\right) \geq\left(N_{S}(h / a)\right)^{2 / r} .
$$

Let $K_{1}=\mathbb{Q}\left(\alpha_{1}, \alpha_{2}\right)$ and let $S_{1}$ be defined for $K_{1}$ in an analogous way to our definition of $S$ for $K$. Let $d_{1}$ be the degree of $K_{1}$ over $\mathbb{Q}$. By (119),

$$
N_{S_{1}}\left(\left(x_{j}-\alpha_{1}\right)\left(x_{j}-\alpha_{2}\right)\right) \geq\left(N_{S_{1}}(h / a)\right)^{2 / r} .
$$

Put $\lambda=a\left(x_{j}-\alpha_{1}\right), \eta=a\left(x_{j}-\alpha_{2}\right)$, and $\tau=a\left(\alpha_{2}-\alpha_{1}\right)$. Then $\lambda, \eta$, and $\tau$ are in $\mathscr{O}_{K_{1}} \backslash\{0\}$ and

$$
a\left(x-\alpha_{1}\right)=\lambda u_{1} \text { and } a\left(x-\alpha_{2}\right)=\eta u_{2},
$$

where $u_{1}$ and $u_{2}$ are $S_{1}$-units. Then

$$
\lambda u_{1}-\eta u_{2}=\tau,
$$

and each pair of $S_{1}$-units $\left(u_{1}, u_{2}\right)$ determines a unique integer $x$.

If $\left(\beta_{1}, \beta_{2}, \beta_{3}\right)$ is a triple in $\left(\mathscr{O}_{K_{1}} \backslash\{0\}\right)^{3}$ that is $S_{1}$-equivalent to $(\lambda, \eta, \tau)$ then, by $(120)$,

$$
\begin{aligned}
N_{S_{1}}\left(\beta_{1} \beta_{2} \beta_{3}\right) & \geq N_{S_{1}}(\lambda \eta \tau)\left(N_{S_{1}}\left(a\left(\alpha_{1}-\alpha_{2}\right)\right)\right)^{-3} \\
& \geq\left(N_{S_{1}}(h)\right)^{2 / r}\left(N_{S_{1}}\left(a\left(\alpha_{1}-\alpha_{2}\right)\right)\right)^{-2} .
\end{aligned}
$$

Thus, as in the proof of (110),

$$
\begin{aligned}
N_{S_{1}}\left(\beta_{1} \beta_{2} \beta_{3}\right) & \geq h^{2 d_{1} / r}\left(N_{S_{1}}(a D)\right)^{-2} \\
& \geq h^{2 d_{1} / r}|a D|^{-2 d_{1}} .
\end{aligned}
$$

We now complete the proof by appealing to Lemmas 7 and 8 as we did for the proof of Theorem 4. Here we make use of the fact that $d_{1} \leq r(r-1)$ and $\left|S_{1}\right|+1 \leq d_{1} t+d_{1}+1<r^{2}(t+1)$.

\section{REFERENCES}

1. Y. Amice, Les nombres p-adiques, Presses Univ. France, 1985.

2. E. Bombieri, On the Thue-Siegel-Dyson theorem, Acta Math. 148 (1982), 255-296.

3. __ On the Thue-Mahler equation, Diophantine Approximation and Transcendence Theory (Seminar, Bonn 1985) (G. Wüstholz, ed.), Lecture Notes in Math., vol. 1290, SpringerVerlag, Berlin, Heidelberg, New York, 1987, pp. 213-243.

4. E. Bombieri and J. Mueller, On effective measures of irrationality for $\sqrt[r]{\frac{a}{b}}$ and related numbers, J. Reine Angew. Math. 342 (1983), 173-196.

5. E. Bombieri and W. M. Schmidt, On Thue's equation, Invent. Math. 88 (1987), 69-81. 
6. N. G. de Bruijn, On the number of positive integers $\leq x$ and free of prime factors $>y$, Nederl. Akad. Wetensch. Proc. Ser. A 54 (1951), 50-60.

7. J. H. H. Chalk and R. A. Smith, Sándor's theorem on polynomial congruences and Hensel's lemma, C. R. Math. Rep. Acad. Sci. Canada 4 (1982), 49-54.

8. S. Chowla, Contributions to the analytic theory of numbers. II, J. Indian Math. Soc. 20 (1933), 120-128.

9. P. Erdös and K. Mahler, On the number of integers which can be represented by a binary form, J. London Math. Soc. 13 (1938), 134-139.

10. P. Erdös, C. L. Stewart, and R. Tijdeman, Some diophantine equations with many solutions, Compositio Math. 66 (1988), 37-56.

11. J. H. Evertse, On the equation $a x^{n}-b y^{n}=c$, Compositio Math. 47 (1982), 289-315.

12. __ Upper bounds for the numbers of solutions of diophantine equations, M.C.-Tract 168, Centre of Mathematics and Computer Science, Amsterdam, 1983.

13. __, On equations in S-units and the Thue-Mahler equation, Invent. Math. 75 (1984), 561-584.

14. J. H. Evertse and K. Györy, Thue-Mahler equations with a small number of solutions, J. Reine Angew. Math. 399 (1989), 60-80.

15. __ Effective finiteness results for binary forms with given discrminant, Compositio Math. 79 (1991), 169-204.

16. __ Thue inequalities with a small number of solutions (to appear).

17. J. H. Evertse, K. Györy, C. L. Stewart, and R. Tijdeman, On S-unit equations in two unknowns, Invent. Math. 92 (1988), 461-477.

18. G. H. Hardy and E. M. Wright, An introduction to the theory of numbers, 5th ed., Oxford Univ. Press, 1979.

19. M. N. Huxley, A note on polynomial congruences, Recent Progress in Analytic Number Theory, Vol. 1 (H. Halberstam and C. Hooley, eds.), Academic Press, London, 1981, pp. 193-196.

20. S. Lang, Integral points on curves, Inst. Hautes Études Sci. Publ. Math. 6 (1960), 27-43.

21. D. Lewis and K. Mahler, Representation of integers by binary forms, Acta Arith. 6 (1961), 333-363.

22. J. H. Loxton and R. A. Srnith, On Hua's estimate for exponential sums, J. London Math. Soc. (2) 26 (1982), 15-20.

23. K. Mahler, Zur Approximation algebraischer Zahlen. II. Uber die Anzahl der Darstellungen ganzer Zahlen durch Binärformen, Math. Ann. 108 (1933), 37-55.

24. __ On the lattice points on curves of genus 1, Proc. London Math. Soc. (2) 39 (1935), 431-466.

25. __ An inequality for the discriminant of a polynomial, Michigan Math. J. 11 (1964), 257-262.

26. __ On Thue's theorem, Math. Scand. 55 (1984), 188-200.

27. L. J. Mordell, Diophantine equations, Academic Press, London and New York, 1969.

28. P. Moree and C. L. Stewart, Some Ramanujan-Nagell equations with many solutions, Nederl. Akad. Wetensch. Proc. Ser. A (N.S.) 1 (1990), 465-472.

29. J. Mueller, and W. M. Schmidt, Thue's equation and a conjecture of Siegel, Acta Math. 160 (1988), 207-247.

30. T. Nagell, Généralisation d'un theórème de Tchebicheff, J. Math. 8 (1921), 343-356.

31. M. Newman, Integral matrices, Pure and Appl. Math. (S. Eilenberg and P. A. Smith, eds.), vol. 45, Academic Press, New York, 1972.

32. O. Ore, Anzahl der Wurzeln höherer Kongruenzen., Norsk Matematisk Tidsskrift, 3 Aagang, Kristiana (1921), 343-356.

33. G. Sándor, Uber die Anzahl der Lösungen einer Kongruenz, Acta. Math. 87 (1952), 13-17. 
34. W. M. Schmidt, Thue equations with few coefficients, Trans. Amer. Math. Soc. 303 (1987), 241-255.

35. C. L. Siegel, Die Gleichung $a x^{n}-b y^{n}=c$, Math. Ann. 114 (1937), 57-68.

36. J. H. Silverman, Representation of integers by binary forms and the rank of the Mordell-Weil group, Invent. Math. 74 (1983), 281-292.

37. _- Integer points on curves of genus 1, J. London Math. Soc. (2) 28 (1983), 1-7.

38. __ The arithmetic of elliptic curves, Graduate Texts in Math., vol. 106, Springer-Verlag, New York, 1986.

Department of Pure Mathematics, The University of Waterloo, Waterloo, Ontario CANADA N2L $3 \mathrm{Gl}$ 